\title{
Establishment of regulatory elements during erythro-megakaryopoiesis identifies hematopoietic lineage-commitment points
}

\author{
Elisabeth F. Heuston' ${ }^{1}$, Cheryl A. Keller², Jens Lichtenberg' ${ }^{1}$, Belinda Giardine², Stacie M. Anderson ${ }^{3}$, NIH
} Intramural Sequencing Center ${ }^{4}$, Ross C. Hardison ${ }^{2}$ and David M. Bodine ${ }^{7^{*}}$

\begin{abstract}
Background: Enhancers and promoters are cis-acting regulatory elements associated with lineage-specific gene expression. Previous studies showed that different categories of active regulatory elements are in regions of open chromatin, and each category is associated with a specific subset of post-translationally marked histones. These regulatory elements are systematically activated and repressed to promote commitment of hematopoietic stem cells along separate differentiation paths, including the closely related erythrocyte (ERY) and megakaryocyte (MK) lineages. However, the order in which these decisions are made remains unclear.

Results: To characterize the order of cell fate decisions during hematopoiesis, we collected primary cells from mouse bone marrow and isolated 10 hematopoietic populations to generate transcriptomes and genome-wide maps of chromatin accessibility and histone $\mathrm{H} 3$ acetylated at lysine 27 binding (H3K27ac). Principle component analysis of transcriptional and open chromatin profiles demonstrated that cells of the megakaryocyte lineage group closely with multipotent progenitor populations, whereas erythroid cells form a separate group distinct from other populations. Using H3K27ac and open chromatin profiles, we showed that $89 \%$ of immature MK (iMK)-specific active regulatory regions are present in the most primitive hematopoietic cells, $46 \%$ of which contain active enhancer marks. These candidate active enhancers are enriched for transcription factor binding site motifs for megakaryopoiesis-essential proteins, including ERG and ETS1. In comparison, only $64 \%$ of ERY-specific active regulatory regions are present in the most primitive hematopoietic cells, $20 \%$ of which containing active enhancer marks. These regions were not enriched for any transcription factor consensus sequences. Incorporation of genome-wide DNA methylation identified significant levels of de novo methylation in iMK, but not ERY.
\end{abstract}

Conclusions: Our results demonstrate that megakaryopoietic profiles are established early in hematopoiesis and are present in the majority of the hematopoietic progenitor population. However, megakaryopoiesis does not constitute a "default" differentiation pathway, as extensive de novo DNA methylation accompanies megakaryopoietic commitment. In contrast, erythropoietic profiles are not established until a later stage of hematopoiesis, and require more dramatic changes to the transcriptional and epigenetic programs. These data provide important insights into lineage commitment and can contribute to ongoing studies related to diseases associated with differentiation defects.

Keywords: Hematopoiesis, Megakaryopoiesis, Erythropoiesis, ATAC-Seq, RNA-Seq, ChIP-Seq, Lineage commitment, Epigenetics

\footnotetext{
*Correspondence: tedyaz@nhgri.nih.gov

${ }^{1}$ NHGRI Hematopoiesis Section, GMBB, Bethesda, MD, USA

Full list of author information is available at the end of the article
} 


\section{Background}

Hematopoiesis is the process by which proliferating hematopoietic stem cells (HSC) undergo continued transcriptional and epigenetic changes associated with lineage-restriction and cell-specific function, giving rise to all of the cell types in the hematopoietic system. Classical models of hematopoiesis describe a series of progressively restricted cell fate decisions in which HSC give rise to multipotent progenitors (MPP), which in turn give rise to common myeloid progenitors (CMP) and common lymphoid progenitors (CLP). In myelopoiesis, CMP differentiate into granulocyte-macrophage progenitors (GMP), from which mature granulocytes and monocytes are generated, and megakaryocytic erythroid progenitors (MEP), which have classically been described as the common erythrocyte and megakaryocyte progenitor [1-4].

Erythro-megakaryopoiesis describes the subset of cell fate decisions associated with the production of erythroblasts (ERY; erythropoiesis) and megakaryocytes (MK; megakaryopoiesis). The process of erythroid and megakaryocyte commitment is accompanied by the substitution of GATA1 for GATA2 at key chromatin regulatory sites to increase expression of linage-specific transcription factors including FLI1 and ETS in the MK lineage, and KLF1 and GATA1 in the ERY lineage [5-8]. Traditional hierarchical models predict that ERY and MK lineages are derived from a homogeneous population of bipotential MEP (Fig. 1a) [9]. However, there is mounting evidence that the MK program is established prior to the emergence of erythroid cells. Several studies have noted transcriptional and immunophenotypic similarities between HSC and MK populations [10-13]. Singlecell transcriptional studies of human MEP demonstrated that traditionally defined MEP included erythroid- and megakaryocyte-committed cells mixed with a small compartment of bipotential intermediates [14]. These studies have led to revised models where many MK are derived from a multipotent stem cell, while others can be generated from CMP and MEP intermediates [15]. While MK appears to have multiple origins, in these revised models the erythroid lineage appears to remain a single branch downstream of the CMP and formed through the MEP.

Determining when lineage specification occurs is important for understanding mechanisms behind diseases with maturation defects. These diseases include anemias, thrombocytopenias, neutropenias, myelodysplastic and myeloproliferative disorders, and hematologic malignancies [16-20]. Fluorescence-activated cell sorting and next-generation sequencing technologies make it possible to generate transcriptome profiles (RNA-Seq), genome-wide profiles of chromatin accessibility (ATAC-Seq), histone modifications (ChIP-Seq), and DNA methylation (MBD-Seq) of cells at specific stages of hematopoiesis. As part of the ValIdated Systematic IntegratiON of hematopoietic epigenomes (VISION) project, we are creating comprehensive catalogs of genomic regulatory elements in primary mouse cells to compare and contrast epigenetic regulation in mice with human data from the Encyclopedia of DNA Elements (ENCODE) project and the NIH Roadmap Epigenomics Project [21-24]. VISION also emphasizes bioinformatics modeling and machine learning to predict regulatory interactions which is enabled by the ability to manipulate mouse hematopoiesis at the genetic level.

To characterize the relationships between hematopoietic populations and the changes that accompany erythro-megakaryopoiesis, we enriched 10 primary hematopoietic cell populations from C57BL6 mice for RNA-Seq, ATAC-Seq, ChIP-Seq, and MBD-Seq analysis. These data have been integrated into transcriptional and epigenetic maps to identify candidate regulatory elements in the ERY and MK lineages and to study the establishment and maintenance of these regulatory elements during erythro-megakaryopoiesis. Our results demonstrate that committed megakaryocytes have similar transcriptional and epigenetic profiles to hematopoietic stem and progenitor cells, but do not represent a "default" developmental program. By contrast, the erythroid population is the most dissimilar from hematopoietic stem and progenitor populations and requires more extensive changes to the transcriptional and epigenetic programs to permit erythropoiesis.

\section{Results}

Committed megakaryocyte precursors have similar transcriptional profiles to hematopoietic progenitor cells

RNA-Seq was performed on two biological replicates of total RNA isolated from 10 populations of primary mouse bone marrow separated on the basis of cell surface marker expression (Fig. 1a, b). We defined "expressed" transcripts as having a transcripts per million count $(\mathrm{TPM}) \geq 1$ (Table 1 ). Because our primary megakaryocytes were not treated with thrombopoietin, these cells do not express many of the genes involved in platelet function; we therefore refer to this population as immature MK (iMK).

Unsupervised hierarchical clustering of the individual RNA-Seq replicates showed that lineage-restricted megakaryocytic populations (CFU-MK and iMK) clustered with progenitors (LSK, CMP, GMP, and MEP), while lineage-restricted erythroid (CFU-E and ERY) and granulocyte populations grouped in different clusters (Fig. 2a). We used principal component analysis (PCA) to display relationships among the transcriptomes on a plane representing the largest variance in the datasets. As expected, replicates for each cell population fell closer to each other 


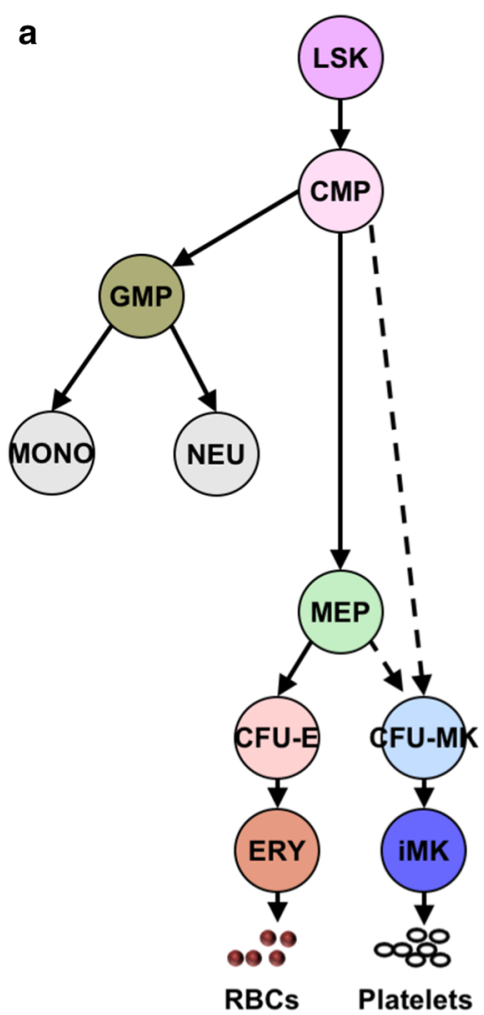

\begin{tabular}{|l|l|l|}
\hline Cell type & Lineage-depletion antibodies & Flow cytometry antibodies \\
\hline LSK & $\begin{array}{l}\text { CD4, CD8, IL7Ra, CD11b, } \\
\text { Ly6g, CD45, Ter119 }\end{array}$ & cKit+, Sca1+ \\
\hline CMP & $\begin{array}{l}\text { CD4, CD8, IL7Ra, CD11b, } \\
\text { Ly6g, CD45, Ter119 }\end{array}$ & $\begin{array}{l}\text { cKit+, Sca1-, CD34+, } \\
\text { CD16/32- }\end{array}$ \\
\hline MEP & $\begin{array}{l}\text { CD4, CD8, IL7Ra, CD11b, } \\
\text { Ly6g, CD45, Ter119 }\end{array}$ & cKit+, Sca1-, CD34-, CD16/32- \\
\hline GMP & $\begin{array}{l}\text { CD4, CD8, IL7Ra, CD11b, } \\
\text { Ly6g, CD45, Ter119 }\end{array}$ & $\begin{array}{l}\text { cKit+, Sca1-, CD34+, } \\
\text { CD16/32+ }\end{array}$ \\
\hline CFU-MK & $\begin{array}{l}\text { CD4, CD8, IL7Ra, CD11b, } \\
\text { Ly6g, CD45 }\end{array}$ & $\begin{array}{l}\text { Ter119-, Sca1-, CD41+, } \\
\text { CD61+, CKit+ }\end{array}$ \\
\hline iMK & $\begin{array}{l}\text { CD4, CD8, IL7Ra, CD11b, } \\
\text { Ly6g, CD45 }\end{array}$ & $\begin{array}{l}\text { Ter119-, Sca1-, CD41+, } \\
\text { CD61+, cKit- }\end{array}$ \\
\hline CFU-E & $\begin{array}{l}\text { CD4, CD8, IL7Ra, CD11b, } \\
\text { Ly6g, CD45 }\end{array}$ & Ter119+, CD44+, FSC(High) \\
\hline ERY & $\begin{array}{l}\text { CD4, CD8, IL7Ra, CD11b, } \\
\text { Ly6g, CD45 }\end{array}$ & Ter119+, CD44+, FSC(Low) \\
\hline MONO & N/A & CD11b+, Ly6g- \\
\hline NEU & N/A & N/A \\
\hline
\end{tabular}

b

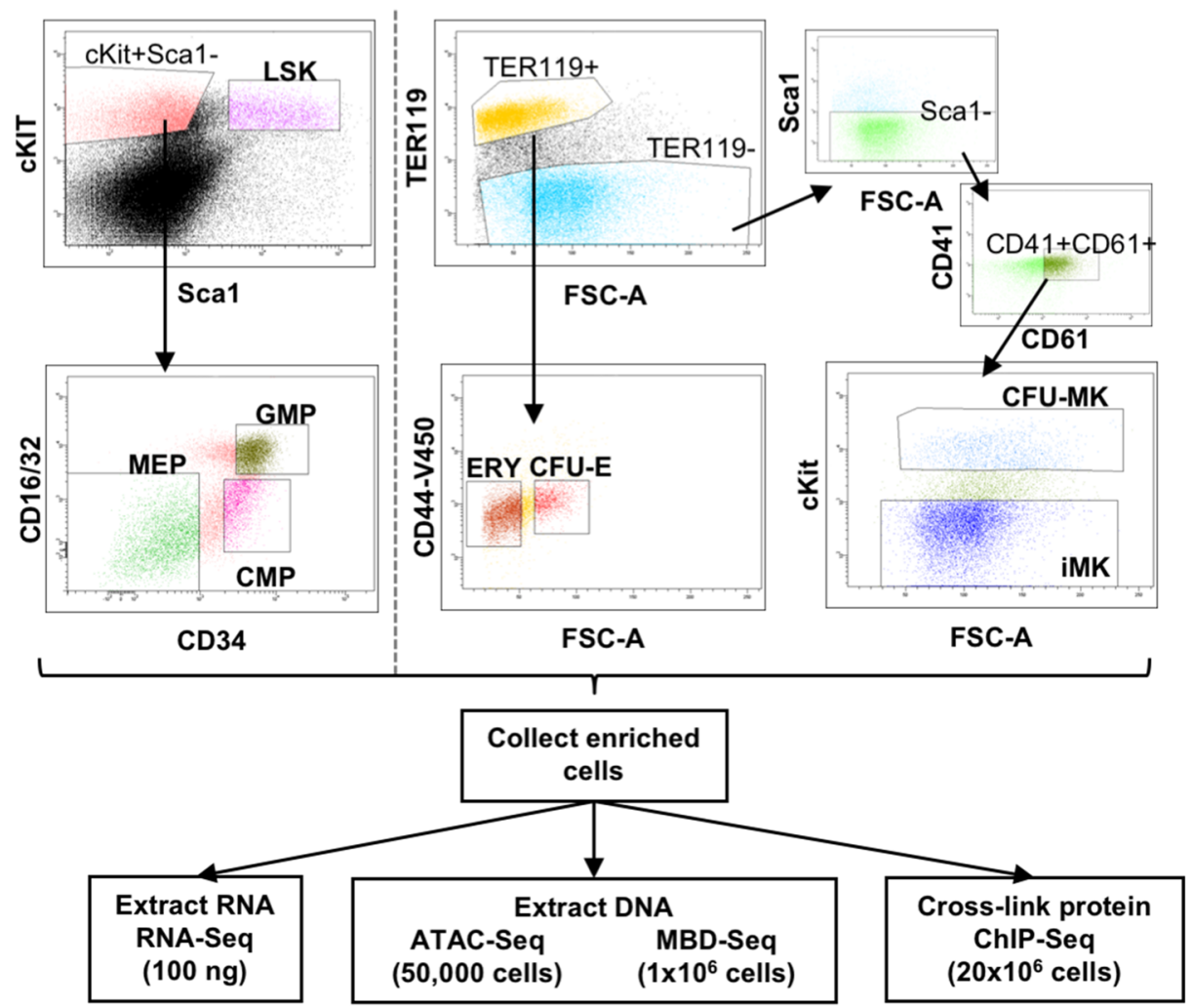

Fig. 1 Primary mouse bone marrow cells isolated via flow cytometry. a Hematopoietic populations assayed in this study. Branches represent lineage-commitment points. Cell surface markers used for flow cytometry enrichment are shown. $\mathbf{b}$ Sort logic is shown for flow cytometry enrichment 
Table 1 Numbers of features ascertained in each cell population

\begin{tabular}{|c|c|c|c|c|}
\hline Population & $\begin{array}{l}\text { Number of transcripts } \\
(\mathrm{TPM} \geq 1)\end{array}$ & $\begin{array}{l}\text { Number of transcripts } \\
\text { (TPM } \geq 10)\end{array}$ & Number of ATAC peaks & $\begin{array}{l}\text { Number } \\
\text { of methylation } \\
\text { peaks }\end{array}$ \\
\hline LSK & 13,066 & 7515 & 64,043 & 33,754 \\
\hline CMP & 12,348 & 7065 & 107,281 & 10,288 \\
\hline CFU-E & 9631 & 3834 & 41,805 & 16,439 \\
\hline ERY & 10,025 & 3525 & 42,260 & 5218 \\
\hline CFU-MK & 11,889 & 7059 & 122,757 & 69,405 \\
\hline iMK & 11,751 & 6848 & 97,463 & 52,754 \\
\hline MEP & 11,574 & 6834 & 76,334 & N/A \\
\hline GMP & 11,749 & 6972 & 112,947 & N/A \\
\hline NEU & 12,250 & 6330 & 44,239 & N/A \\
\hline MONO & 11,857 & 6239 & 53,531 & $\mathrm{~N} / \mathrm{A}$ \\
\hline
\end{tabular}

than to other cell types. Principal component 1 (PC1), which accounted for $30 \%$ of the variance, separated erythroid, monocyte, and neutrophil cells from progenitor cells. PC2, which accounted for $23 \%$ of the total variance, separated erythroid cells from all other cells (Fig. 2b). Strikingly, the transcriptome of the committed MK progenitor, CFU-MK, grouped with progenitor cells, while the iMK grouped closest to this population.

Of the 13,000 transcripts expressed in our multipotent progenitor population (LSK), 81\% were also expressed in the iMK population (Fig. 2c, solid circle); this fraction represents $89 \%$ of all iMK transcripts (Fig. 2c, dotted circle). In contrast, $63 \%$ of transcripts expressed in LSK were expressed in ERY, representing $82 \%$ of all ERY transcripts. This fraction is significantly smaller than that observed for the iMK population (proportion test $p$ value $<0.001$ ) (Fig. 2c, Table 2). Of the almost 12,000 expressed CMP transcripts, 1930 (16\%) were expressed in iMK but not in ERY, whereas only 294 (2\%) were expressed in ERY but not in iMK (proportion test $p$ value $<0.001$ ) (Fig. 2d, Table 2). These results show that cellular transcriptomes of the megakaryocytic lineage are similar to those of multilineage progenitor cells, whereas erythroid cells repress the multilineage transcriptome.

\section{Committed megakaryocytes have a similar chromatin accessibility profile to hematopoietic progenitor cells}

We generated maps of accessible chromatin regions using the Assay for Transposase-Accessible Chromatin (ATAC) on the same populations of primary mouse bone marrow cells used for transcriptional profiling. Unsupervised hierarchical clustering of the average ATAC-Seq signal in each peak region showed that, like the transcriptional profiles, the ATAC profiles of iMK and CFU-MK clustered with LSK, CMP, and GMP, while the ATAC profiles of the erythroid, neutrophil, and monocyte populations clustered separately (Fig. 3a). These groups of committed erythroid cells and committed granulocytes were also observed in the PCA, while CFU-MK and iMK again grouped with the multilineage progenitor cells (Fig. 3b). Unlike the transcriptional profiles, profiles of MEP chromatin accessibility clustered with erythroid cells rather than with the multilineage progenitors.

Of 64,000 ATAC-Seq peaks in LSK, more than 95\% of these were present in the set of $\sim 100,000$ CMP peaks (Fig. 3c, Table 1). Similarly, 76\% ( 48,695) of LSK ATACSeq peaks were present in the set of $\sim 97,000$ iMK peaks (representing $72 \%$ of all iMK peaks) (Fig. 3c, Table 1). In contrast, only $33 \%(\sim 21,000)$ of LSK ATAC-Seq peaks were present in the set of $\sim 42,000$ ERY peaks (representing $50 \%$ of all ERY peaks). While less overlap was observed among CMP, ERY, and iMK ATAC-Seq profiles compared to the overlap observed in RNA-Seq profiles, a substantially larger number of peaks were shared between iMK and CMP than between ERY and CMP (Fig. 3d). We conclude that iMK maintains much of the transposase-accessible chromatin that is established in LSK, while erythropoiesis involves significant loss of accessibility in chromatin that was open in progenitors, implying substantial compaction of chromatin during erythropoiesis.

\section{Megakaryocytic regulatory elements are established in multipotent progenitor cells, while many erythroid regulatory elements are established in more differentiated cells}

Active enhancers and promoters are marked by the presence of histone H3 acetylated at lysine 27 (H3K27ac) [25]. We generated genome-wide maps of candidate enhancers and promoters in ERY and iMK by performing ChIP-Seq of H3K27ac. Using four replicates for each population, we identified 8566 ERY and 12,594 iMK H3K27ac peaks 
a

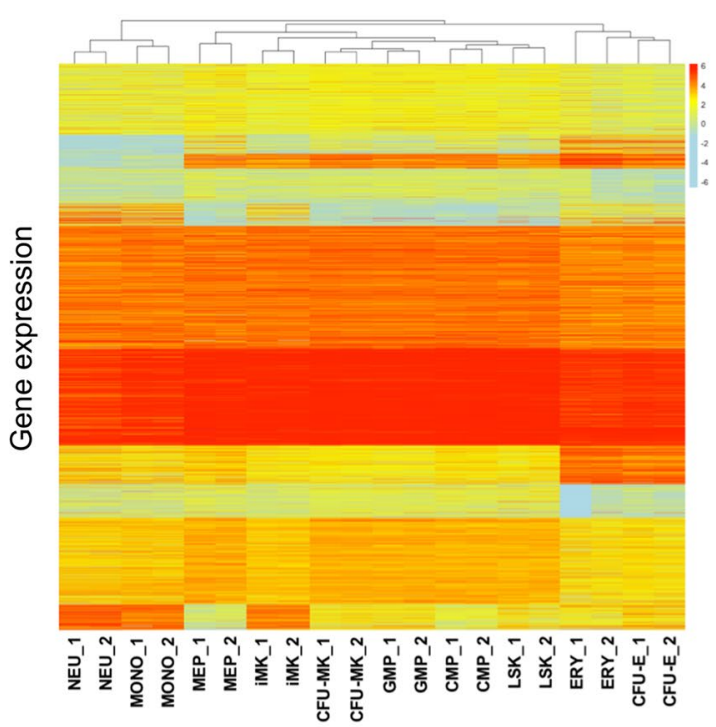

b

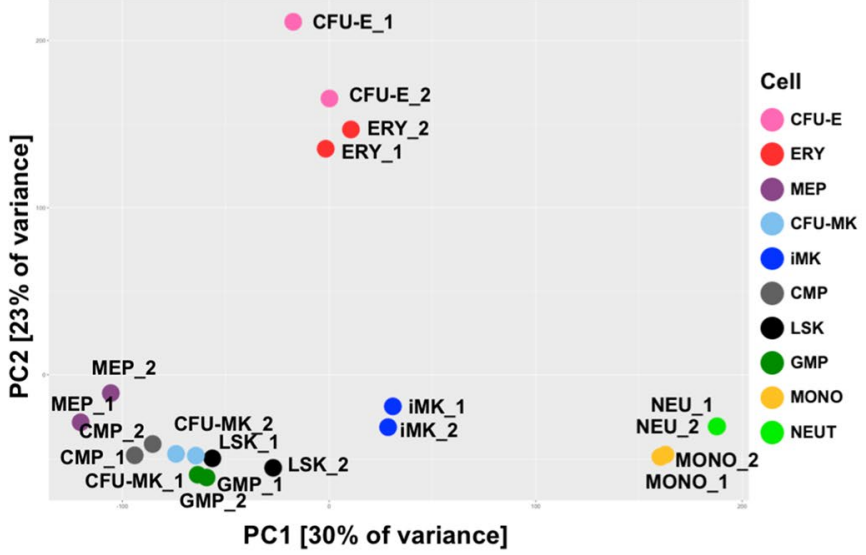

c

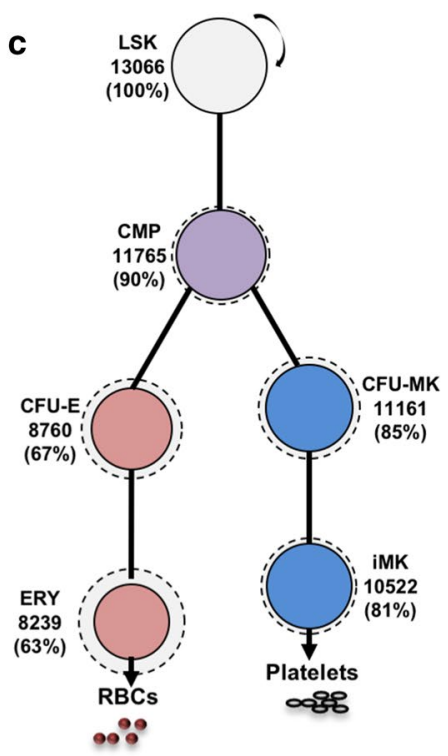

d

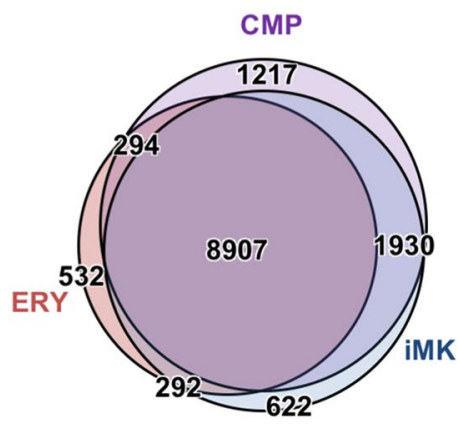

Fig. 2 Transcriptional profiles of hematopoietic populations. a Unsupervised hierarchical clustering analysis of RNA-Seq-derived transcriptomes. b Principle component analysis plotting PC1 versus PC2. c Expressed transcripts in LSK and maintained throughout erythro-megakaryopoiesis. Solid circle size is proportional to the percentage of expressed transcripts maintained from the LSK, dotted circle is proportional to the percentage of all transcripts in the cell relative to the number maintained from LSK, and line length is inversely proportional to percentage of expressed transcripts maintained from the progenitor. The number and percentage of maintained LSK transcripts is shown. $\mathbf{d}$ Proportional Venn diagram indicating overlap of expressed genes between CMP, ERY, and iMK populations

(Fig. 4a, b). Since active enhancers and promoters are almost always in regions of open chromatin, we generated a set of high-confidence active regulatory elements (AREs) by overlapping the H3K27ac peaks with ATACSeq peaks. We found that over 95\% of H3K27ac peaks overlapped with ATAC regions. We identified 2098 ERY-specific and 6386 iMK-specific AREs, and 5989 common AREs shared by both cell types (Fig. 4b). We assigned AREs to candidate target genes using the closest transcriptional start site (TSS), regardless of distance or activation state. This method assigned the majority of
AREs to actively expressed candidate target genes: $82 \%$ of ERY-specific AREs, 89\% of iMK-specific AREs, and 93\% of shared AREs (Table 3). To infer the stage at which ERY or iMK AREs appear (inferred AREs), we intersected the sets of ERY and iMK AREs with the ATAC-Seq peaks of progenitor cells (Fig. 4c). Between 80 and $90 \%$ of these inferred AREs were associated with an actively transcribed gene (Table 3). Over $98 \%$ of shared ERY and iMK AREs were present in LSK. 89\% of iMK-specific AREs were present in LSK, while $97 \%$ were present in CMP. To calculate the significance of the overlap, we performed 
Table 2 Number of transcripts with maintained expression at subsequent stages of hematopoiesis

\begin{tabular}{lccc}
\hline & Expressed in LSK and CMP & $\begin{array}{c}\text { Expressed in LSK, CMP, } \\
\text { and committed progenitor }\end{array}$ & $\begin{array}{l}\text { Expressed in LSK, CMP, } \\
\text { committed progenitor, } \\
\text { and mature cell }\end{array}$ \\
\hline ERY & & & 8239 \\
\# Expressed since LSK & 11,765 & 8760 & 63 \\
\% Expressed since LSK & 90 & 67 & 94 \\
\% Expressed from immediate progenitor & 90 & 74 & 10,522 \\
iMK & 11,765 & 11,161 & 81 \\
\# Expressed since LSK & 90 & 85 & 94 \\
\% Expressed since LSK & 90 & 95 & \\
\% Expressed from immediate progenitor & & & 95 \\
\hline
\end{tabular}

permutation tests with the R package "regioneR" [26]. This analysis demonstrated that the observed number of overlaps was statistically higher than one would expect by random chance (LSK $p$ value 0.002 , CMP $p$ value 0.002 ) (Fig. 4d). In contrast, while 64\% of ERY-specific AREs had already been established in LSK, this portion is significantly lower compared to $89 \%$ of iMK-specific AREs ( $p$ value 0.002). More ERY-specific AREs (84\%) were present in CMP, but this was significantly lower than the $97 \%$ of iMK-specific AREs ( $p$ value 0.002 ). Approximately $14 \%$ of ERY-specific AREs were established de novo in CFU-E ( $p$ value 0.002 ), whereas only $1 \%$ of iMK-specific AREs are established de novo in CFU-MK ( $p$ value 0.002) (Fig. 4d). We conclude that the regulatory element profiles showed a greater amount of lineage-specific activation in ERY than in iMK, similar to what was observed for the transcriptional profiles.

Poised enhancers are defined as regions of accessible chromatin that contain histone H3 monomethylated at lysine 4 (H3K4me1), but they can be distinguished from active enhancers by the absence of H3K27 acetylation [25]. To determine whether the inferred AREs in progenitor cells were poised (H3K4me1 only) or active (H3K4me1 and H3K27ac) in LSK, we compared our data with the indexing-first (iChIP) H3K4me1 and H3K27ac regions identified by Lara-Astiaso et al. [27]. We accessed iChIP sequencing reads from Gene Omnibus (GSE60103) and performed genome alignment and peak calling using our pipeline (see Materials and Methods). This analysis identified 62,849 H3K4me1 and 16,098 H3K27ac peaks in the iChIP LSK set. Approximately $57 \%$ of the LSK-established ERY-specific and 41\% iMK-specific AREs overlapped with H3K4me1 peaks but not H3K27ac peaks, classifying them as poised enhancers (Fig. 5a, Additional file 1: Fig. S1). Approximately $20 \%$ of LSK-established ERY-specific and $46 \%$ of LSK-established iMK-specific AREs overlapped with both H3K4me1 and H3K27ac, classifying them as active enhancers. In comparison,
15 and 79\% of LSK-accessible shared AREs were classified as poised and active enhancers, respectively. $21 \%$ of LSK-established ERY-specific and 9\% of LSK-established iMK-specific AREs did not overlap with either H3K4me1 or H3K27ac peaks, and were therefore classified as open AREs. As validation, 23 of our ERY AREs overlapped with a set of 39 enhancers validated by luciferase assays [28], while none were found in the set of open AREs. Permutations tests using 500 iterations of randomized peak overlaps demonstrated that these intersections were statistically higher than expected by chance alone (Fig. 5b).

To visualize the integration of RNA-Seq, ATAC-Seq, and ChIP-Seq profiles, we examined several genes significant for ERY and iMK maturation (Fig. 6, Additional file 2: Fig. S2). The gene Slc4a1, encoding the anion transporter BAND3, is strongly induced during erythroid maturation. This induction was accompanied by the ERYspecific induction of transposase-accessible chromatin around the promoter and $3^{\prime}$ end of the gene, as well as in the upstream noncoding gene Bloodlinc (Fig. 6a). Chromatin in these regions was also was modified with $\mathrm{H} 3 \mathrm{~K} 27 \mathrm{ac}$ and $\mathrm{H} 3 \mathrm{~K} 4 \mathrm{me} 1$, indicative of active elements (Fig. 6a). The closely linked gene Slc25a39, encoding a constitutively expressed mitochondrial carrier protein, was in accessible chromatin in all the examined cell types (Fig. 6a). In contrast, the Itgb3 gene (encoding the surface marker glycoprotein IIIa or CD61) was expressed and in regions of open chromatin in LSK, CMP, and MK-committed populations, but was repressed and in non-accessible chromatin regions in erythroid cells (Fig. 6b).

\section{Differing properties of ERY- and iMK-specific AREs}

We plotted the proximity of AREs established during hematopoiesis to the closest TSS. AREs within $1 \mathrm{~Kb}$ of the TSS were defined as candidate promoter elements (cPE), and AREs outside of this region were defined as candidate enhancer elements (cEE). Based on these criteria, approximately 85\% (1800) of ERY-specific and 55\% 


\section{a}

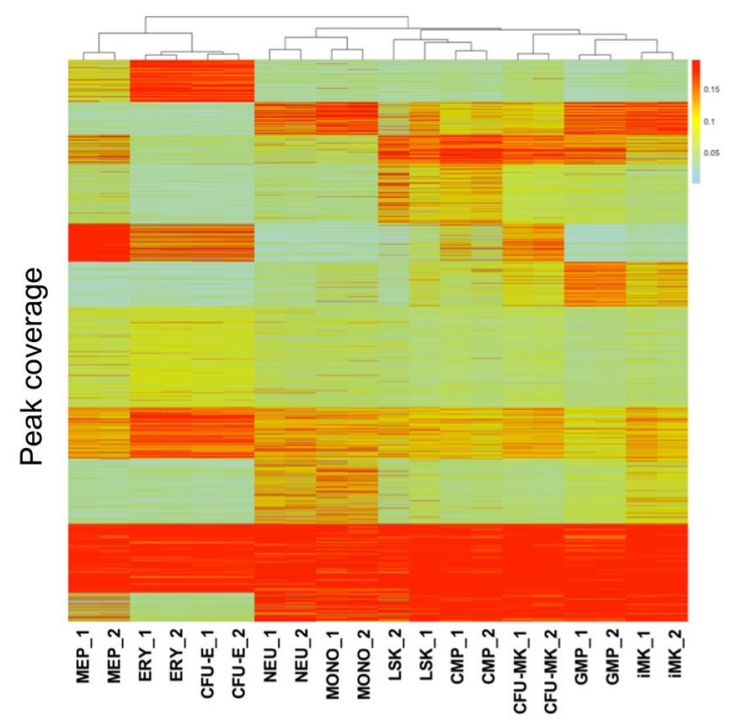

b

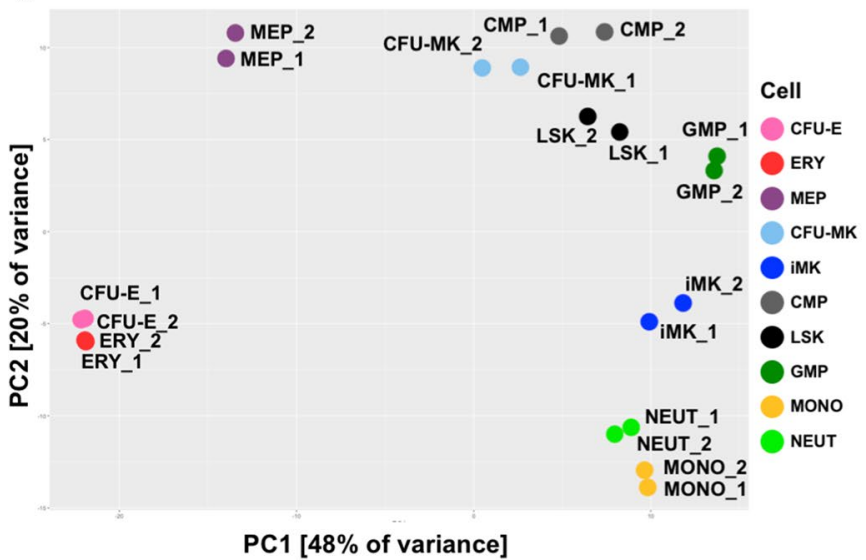

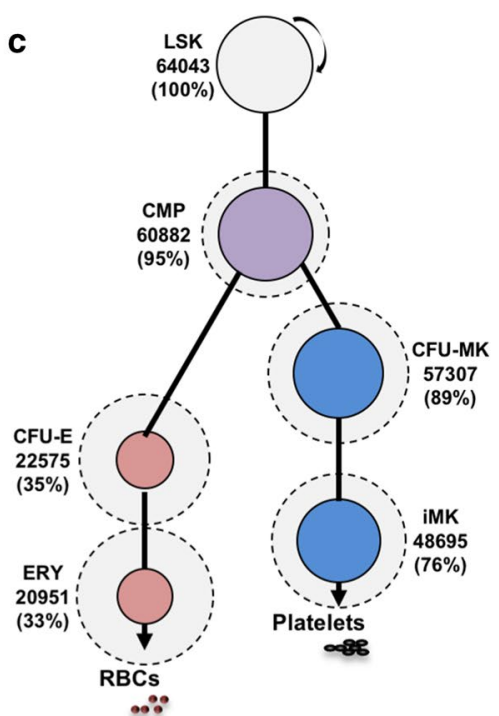

d

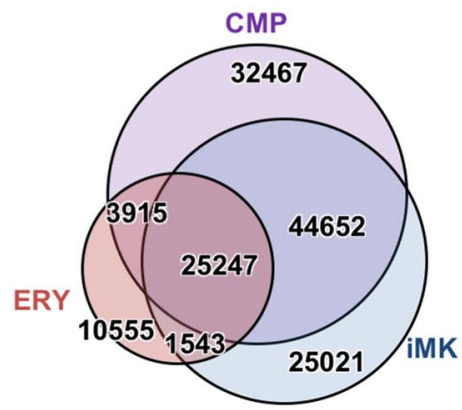

Fig. 3 ATAC-Seq profiles of hematopoietic populations. a Unsupervised hierarchical clustering analysis of ATAC-Seq-derived peak profiles. $\mathbf{b}$ Principle component analysis plotting PC1 versus. c ATAC-Seq peaks in LSK and maintained throughout erythro-megakaryopoiesis. Circle size is proportional to the percentage of peaks maintained from the LSK, dotted circle is proportional to the percentage of all peaks in the cell relative to the number maintained from LSK, and line length is inversely proportional to percentage of peaks maintained from the progenitor. The number and percentage of maintained LSK peaks is shown. $\mathbf{d}$ Proportional Venn diagram indicating overlap of ATAC-Seq peaks between CMP, ERY, and iMK populations

(3500) of iMK-specific AREs established during differentiation were categorized as $\mathrm{CPE}$ (Fig. 7a), with primitive cells having more cPE than committed cells. We also observed that cEE established de novo during differentiation tended to form closer to the TSS in both ERY and iMK populations. Together these data demonstrate that ERY-specific AREs established early in hematopoiesis are more likely to be $\mathrm{cPE}$, whereas a substantially larger fraction of iMK-specific AREs are comparatively more likely to be cEE.
In addition to AREs established at different stages of hematopoiesis, we plotted the proximity of active, poised, and inactive AREs to the nearest TSS. As with ERYspecific AREs established during differentiation, ERYspecific active, poised, and inactive AREs were almost exclusively categorized as cPE (Fig. 7b). While iMKspecific inactive AREs are almost exclusively defined as CPE, approximately $20 \%$ of active and poised AREs were classified as cEE. We conclude that LSK-established ERYspecific AREs are more likely to be candidate promoters, 


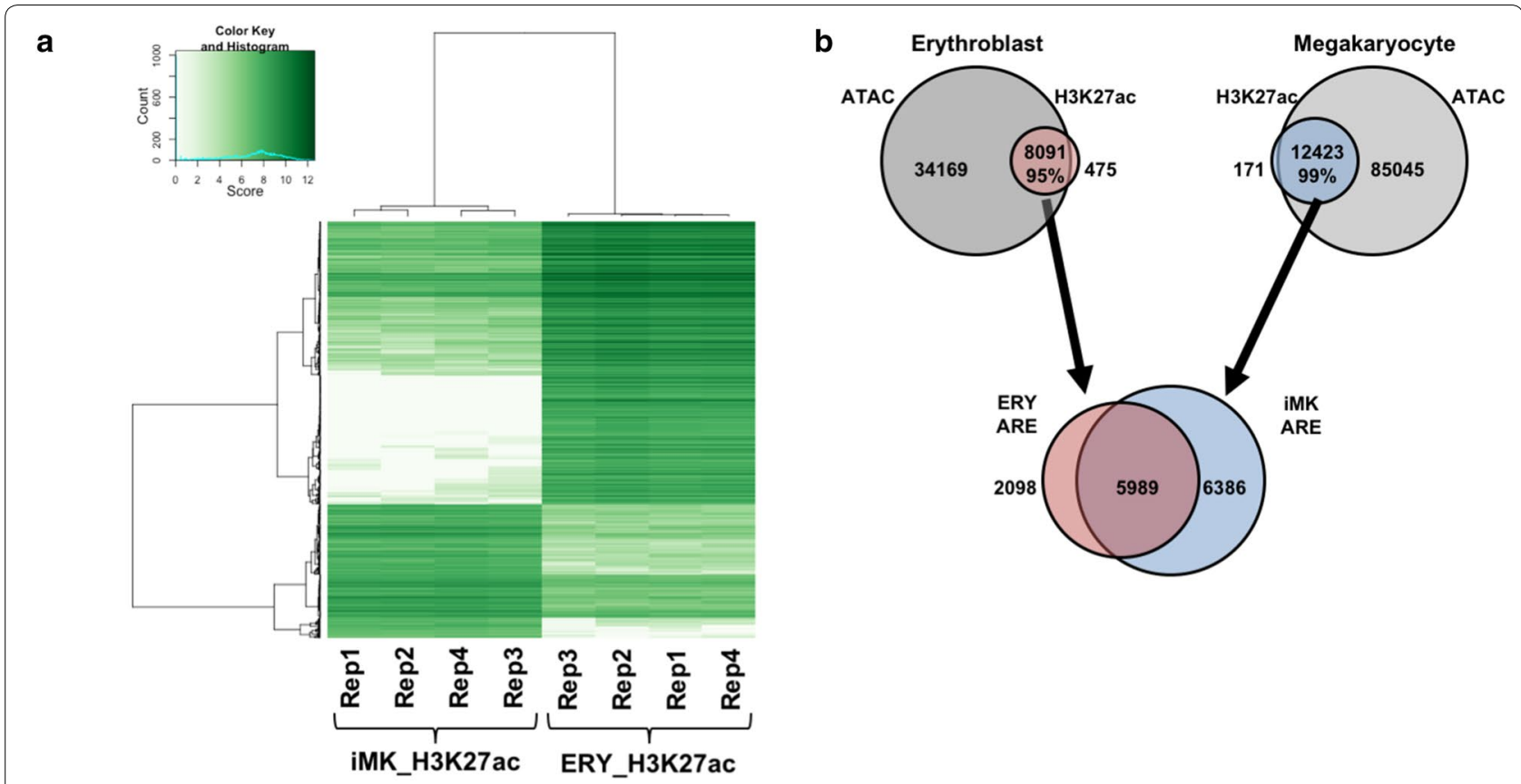

C
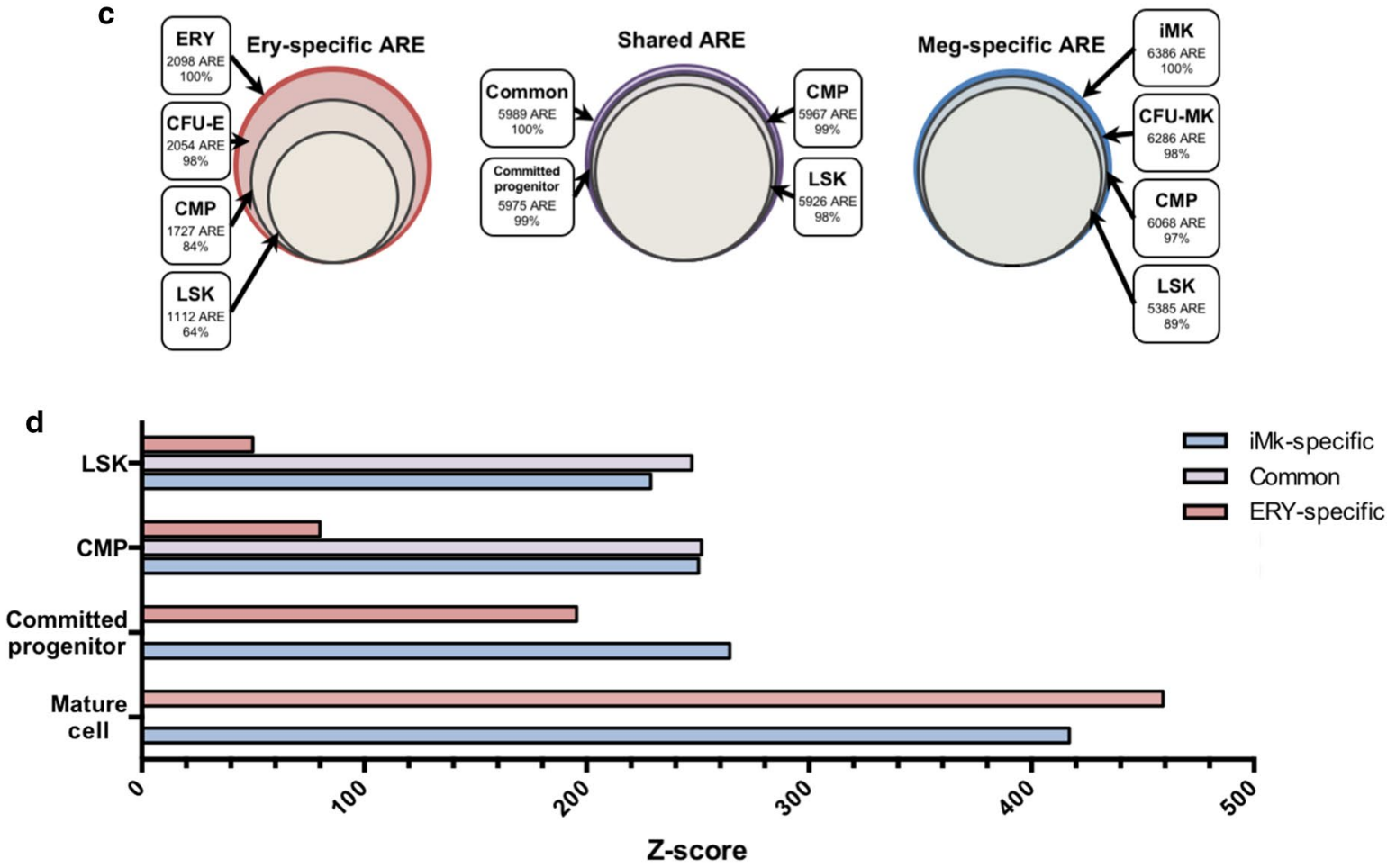

Fig. 4 Establishment of ERY and iMK AREs throughout hematopoiesis. a Heatmap comparing H3K27ac immunoprecipitation peaks in ERY and iMK samples. Calculations were performed using the R package DiffBind (v2.2.6). b Active regions are defined as the intersection of ATAC and H3K27ac peaks in ERY and iMK. c Establishment of open chromatin was defined as intersecting AREs from ERR or iMK with ATAC-Seq peaks in sequentially more primitive cell populations. $\mathbf{d}$ Significance of overlap was calculated by randomizing peak positions and calculating random versus expected overlap (500 iterations). Z-score of overlap between AREs and ATAC peaks. Hashed bars indicate $p$ value $>0.05$ 
Table 3 Number of AREs assigned to the closest TSS

\begin{tabular}{|c|c|c|c|c|c|c|c|}
\hline \multirow{2}{*}{$\begin{array}{l}\text { ARE establishment stage } \\
\text { LSK }\end{array}$} & \multirow{2}{*}{$\begin{array}{l}\text { Category } \\
\text { ARES }\end{array}$} & \multicolumn{2}{|c|}{ ERY-specific } & \multicolumn{2}{|c|}{ Common } & \multicolumn{2}{|c|}{ MEG-specific } \\
\hline & & 1112 & & 5926 & & 5385 & \\
\hline & No. genes assigned as ARE targets & 1118 & & 7963 & & 4462 & \\
\hline & No. genes expressed & 940 & $84 \%$ & 7459 & $93 \%$ & 3965 & $89 \%$ \\
\hline \multirow[t]{3}{*}{ CMP } & ARES & 1727 & & 5967 & & 6068 & \\
\hline & No. genes assigned as ARE targets & 1606 & & 7982 & & 4767 & \\
\hline & No. genes expressed & 1294 & $81 \%$ & 7415 & $94 \%$ & 4083 & $86 \%$ \\
\hline \multirow[t]{3}{*}{ Committed progenitor } & AREs & 2054 & & 5975 & & 6286 & \\
\hline & No. genes assigned as ARE targets & 1829 & & 7987 & & 4848 & \\
\hline & No. genes expressed & 1443 & $79 \%$ & 7266 & $90 \%$ & 4238 & $87 \%$ \\
\hline \multirow[t]{3}{*}{ Terminally differentiated cell } & AREs & 2098 & & 5989 & & 6386 & \\
\hline & No. genes assigned as ARE targets & 1853 & & 7998 & & 4890 & \\
\hline & No. genes expressed & 1527 & $82 \%$ & 7464 & $93 \%$ & 4341 & $89 \%$ \\
\hline
\end{tabular}

a

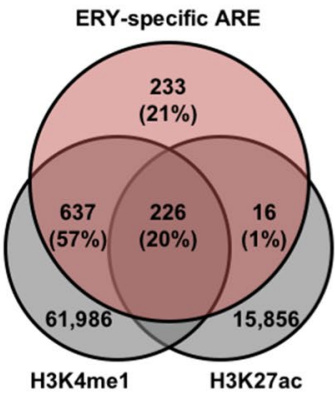

b

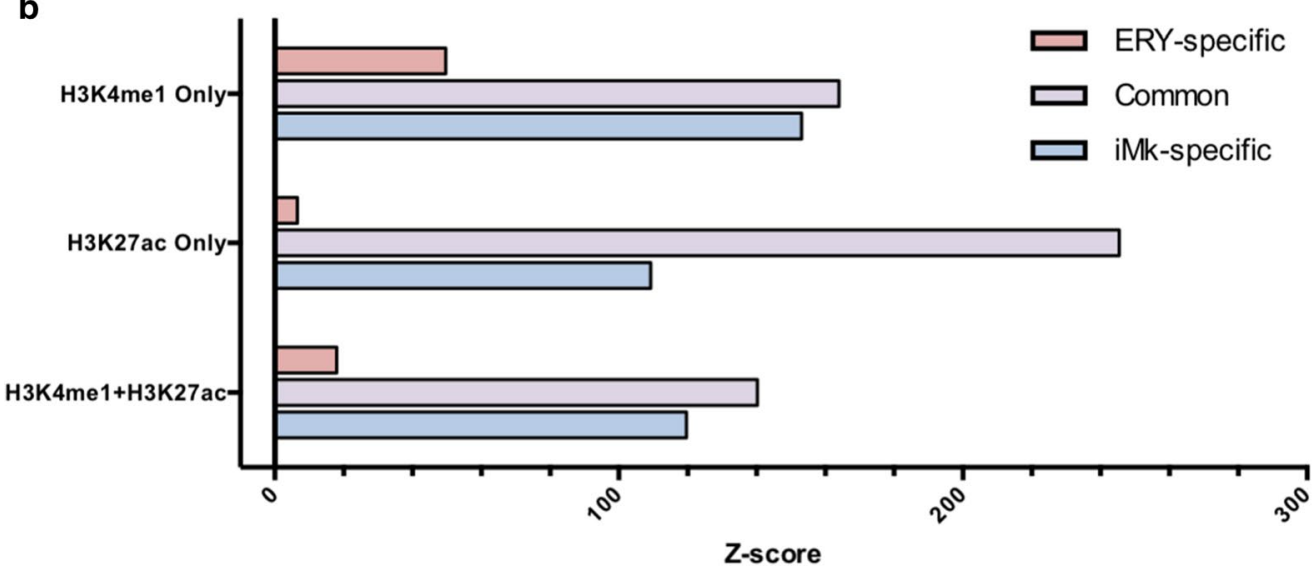

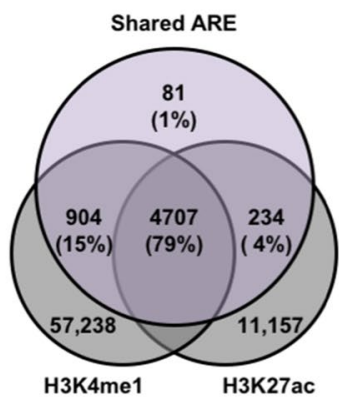

H3K4me1 H3K27ac

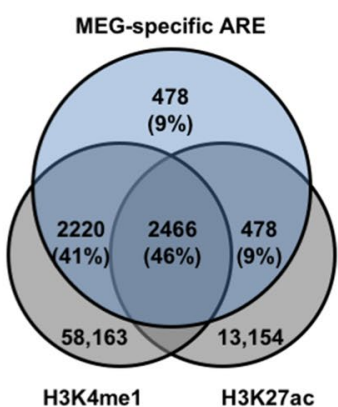

H3K4me1

\section{Z-score}

$9 \%$

$86 \%$

$7 \%$

$89 \%$

Fig. 5 Establishment of active and poised enhancers in LSK. a LSK-accessible cell-specific and shared AREs were compared against the indexing-first H3K4me1 and H3K27ac chromatin immunoprecipitation profiles (Lara-Astiaso et al, Science, 2014). b Z-score of overlap between ARE and iChIP peaks 


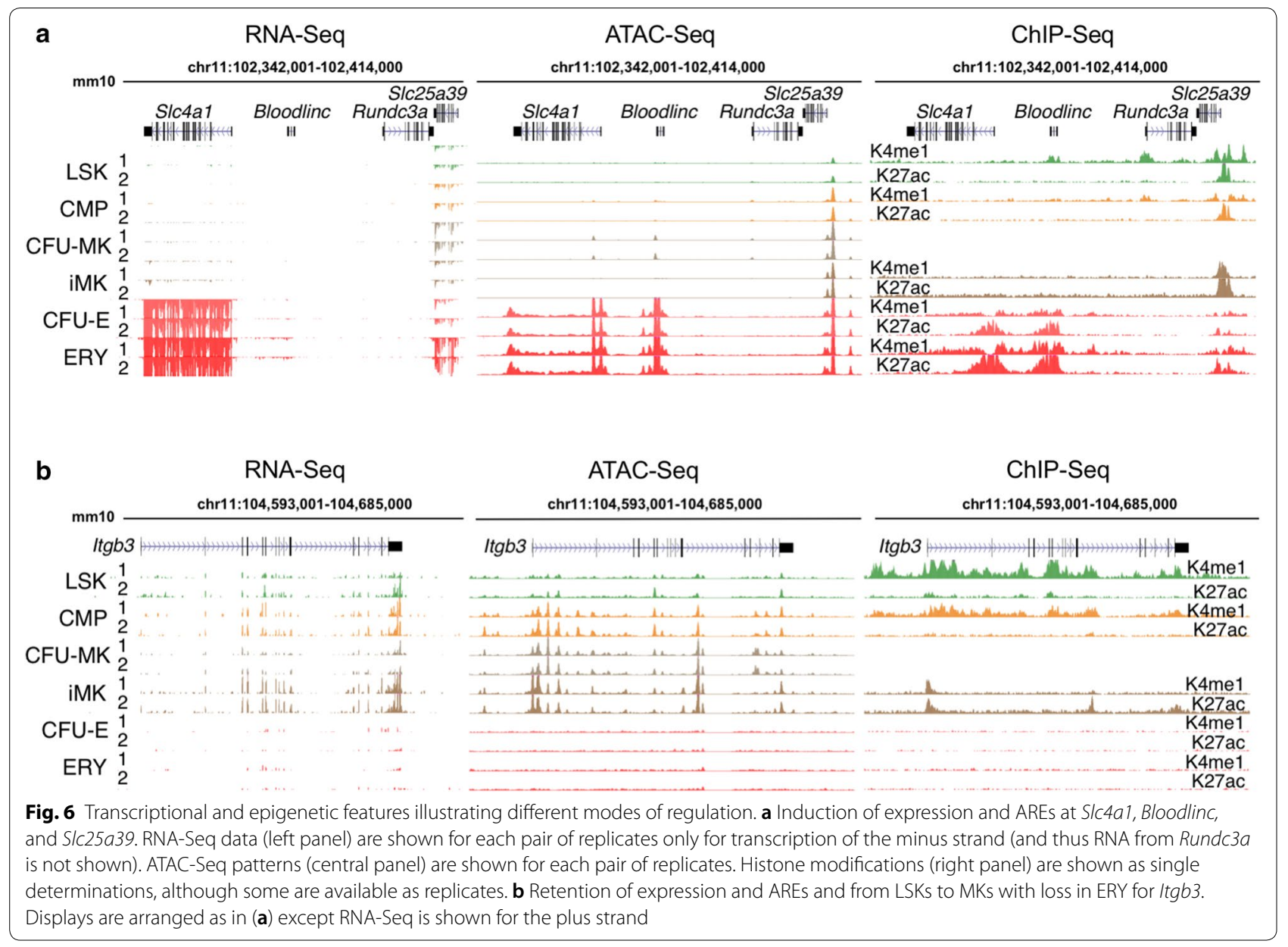

whereas a larger proportion of LSK-established iMK-specific AREs qualify as candidate enhancers.

AREs often contain consensus sequences for transcription factor binding sites that regulate cellular development and lineage specificity. We identified instances of consensus transcription factor binding site motifs (TFBS motifs) that were significantly enriched $(q$-value $\leq 0.05)$ in the set of AREs (Table 4, Additional file 3: Table S1). Poised iMK-specific AREs included motifs for ETS-family proteins FLI1 ( $q$-value 0.029 ), ETS1 ( $q$-value 0.004), and PU.1 ( $q$-value 0.004). In addition to those found in poised iMK-specific AREs, active iMK-specific AREs also had matches to motifs for RUNX1 ( $q$-value 0.046) and RUNX2 ( $q$-value 0.010). ERY-specific AREs established in CMP were enriched for TFBS motifs of the master regulators GATA1, GATA2, and NF-E2 ( $q$-value $\leq 0.03)$ (Table 4). Note that while many TFBS motifs are enriched in AREs present at all stages of hematopoiesis, Table 4 only shows those motifs whose enrichment passes a stringent FDR threshold of $q$-value $\leq 0.05$. In summary, our data suggest that megakaryocytes maintain epigenetic and transcriptional profiles present in the progenitor cell populations, while erythroblasts acquire their distinct profiles later in hematopoiesis.

\section{The megakaryocytic lineage is associated with de novo DNA methylation}

We generated genome-wide DNA methylation maps in LSK, CMP, CFU-E and ERY, and CFU-MK and iMK populations using recombinant Methyl Binding Domain 2 (MBD2) protein to enrich methylated DNA fragments, followed by next-generation sequencing (MBD-Seq) [29]. We identified 33,754 LSK and 10,288 CMP methylation peaks; $86 \%$ of CMP peaks were present in LSK (Table 1). In differentiating erythroid cells, we observed a further loss of the DNA methylation peaks present in CMP accompanied by an additional 9000 de novo methylation peaks in CFU-E. Mature erythroblasts had a global loss of methylation peaks accompanied by additional de novo methylation (Fig. 8a). Megakaryopoiesis was associated with an increase of $\sim 60,000$ de novo methylation peaks in CFU-MK, followed by a loss 


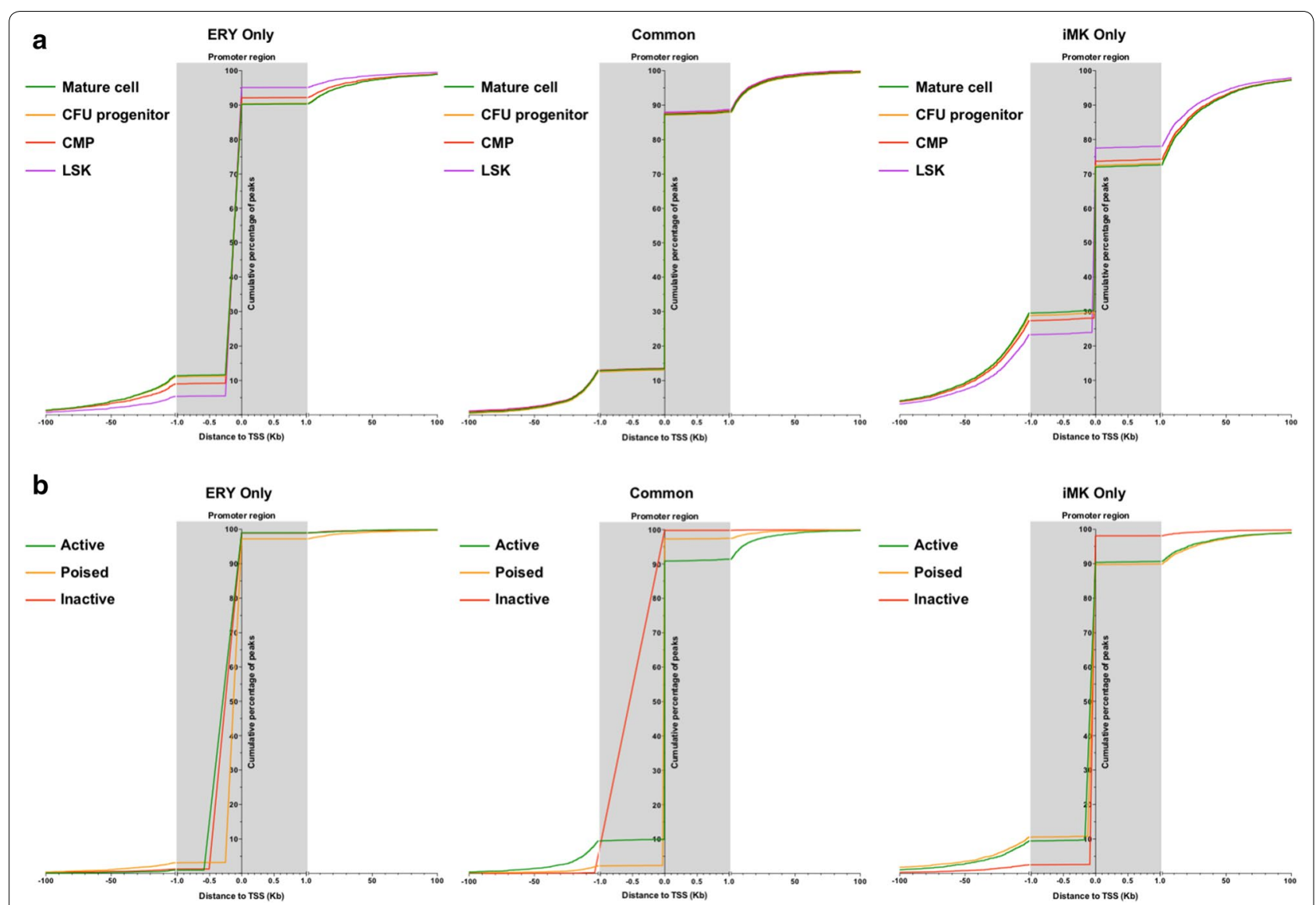

Fig. 7 Distance of ARE to closest TSS. a Distance of LSK-established ARE to the transcriptional start site. $\mathbf{b}$ Distance of active, poised, and inactive ARE to the transcriptional start site

of $\sim 20,000$ of these de novo methylation peaks between CFU-MK and iMK (Fig. 8b). These patterns are illustrated in the transferrin receptor 2 (Tfr2) gene (Fig. 8c).

Because changes in DNA methylation are associated with differences in expression, we compared the overlap of DNA methylation peaks with cell-specific ARE profiles. We found that 43 (2.1\%) of 2098 ERY-specific AREs established in ERY overlapped with ERY DNA methylation peaks (Table 5). 236 (11.5\%) of 2054 ERY-specific AREs established in CFU-E overlapped with CFU-E DNA methylation peaks. In comparison, 789 (12.4\%) of 6368 iMK-specific AREs established in iMK overlapped with iMK DNA methylation peaks, while 1121 (17.8\%) of 6286 iMK-specific AREs established in CFU-MK overlapped with CFU-MK DNA methylation (Table 5).

We compared the overlap of DNA methylation peaks with active, poised, and inactive regions in LSK. We found that nearly $20 \%$ of active and poised ERY-specific regulatory elements overlapped with regions of DNA methylation in LSK, whereas $\sim 8 \%$ of active and poised iMK-specific regulatory elements overlapped with DNA methylation in LSK (Table 6). We conclude that, unlike the ATAC and transcriptional profiles, the methylation profile of the megakaryocytic lineage is acquired during differentiation while erythropoiesis is associated with global DNA demethylation.

\section{Discussion}

It is well known that megakaryocytes and stem cells share a number of molecular features [10, 11]. Both HSC and megakaryocytes have similar dependencies on the thrombopoietin receptor MPL and express CD41 and CD117 (cKit) [10, 11, 30]. HSC and megakaryocytes also have similar dependencies on transcription factors, including expression of RUNX1, GATA2, and EVI1 [11]. Our data suggest that there is a large population of megakaryocyte-primed cells within the multipotent progenitor population (MPP) that shares the chromatin, enhancer/promoter, and transcriptional profiles of the MPP. It is also possible that only a subpopulation of HSC are megakaryocyte-primed, but our data suggest that the majority of MPP share transcriptional and regulatory 
Table 4 Partial list of TFBS motifs enriched in AREs at different stages of hematopoiesis

\begin{tabular}{|c|c|c|c|c|c|c|c|c|}
\hline \multirow{2}{*}{$\begin{array}{l}\text { Transcription } \\
\text { factor }\end{array}$} & \multicolumn{4}{|c|}{ ERY-specific AREs } & \multicolumn{4}{|c|}{ MEG-specific AREs } \\
\hline & LSK (active) & LSK (poised) & CMP & CFU-E & LSK (active) & LSK (poised) & CMP & CFU-MK \\
\hline AP-1 & & & & & & 0.005 & $<0.0001$ & $<0.0001$ \\
\hline $\mathrm{BACH} 1$ & & & & 0.001 & & & & \\
\hline BATF & & & & & & 0.005 & 0.0001 & 0.002 \\
\hline CEBP & & & & & & & 0.001 & 0.001 \\
\hline $\mathrm{CHOP}$ & & & & & & & 0.0001 & 0.001 \\
\hline NF-E2 & & & 0.02 & 0.0002 & & & & \\
\hline EHF & & & & & 0.0001 & 0.005 & $<0.0001$ & $<0.0001$ \\
\hline ELF1 & & & & & 0.0001 & & $<0.0001$ & $<0.0001$ \\
\hline ERG & & & & & 0.0009 & & $<0.0001$ & $<0.0001$ \\
\hline ETS & & & & & 0.0001 & 0.0003 & $<0.0001$ & $<0.0001$ \\
\hline ETS1 & & & & & 0.0008 & & $<0.0001$ & $<0.0001$ \\
\hline PU.1 & & & & & 0.0148 & 0.0039 & $<0.0001$ & $<0.0001$ \\
\hline SpiB & & & & & 0.0004 & 0.0017 & $<0.0001$ & $<0.0001$ \\
\hline FLI1 & & & & & & & $<0.0001$ & $<0.0001$ \\
\hline IRF1 & & & & & 0.0138 & 0.0017 & $<0.0001$ & $<0.0001$ \\
\hline IRF2 & & & & & 0.0052 & & 0.0001 & 0.0005 \\
\hline IRF8 & & & & & 0.0459 & & & \\
\hline RUNX1 & & & & & & & 0.0004 & 0.002 \\
\hline RUNX2 & & & & & & & 0.002 & 0.01 \\
\hline GATA1 & & & 0.03 & 0.01 & & & & \\
\hline GATA2 & & & 0.03 & 0.01 & & & & \\
\hline KLF3 & & & & & & 0.0281 & & \\
\hline SP1 & & & & & & & 0.04 & \\
\hline
\end{tabular}

Benjamini-adjusted $q$-value for the enrichment score is reported

characteristics with those of the megakaryocyte population $[4,15,31-34]$.

The clearest distinction between MPP and megakaryocytes is in their DNA methylation profiles, where de novo methylation increases in CFU-MK but subsequently decreases in iMK [29]. Similar patterns have been observed in muscle and neuronal lineages (reviewed in [35-37]). Our data suggest that de novo methylation is an important step in megakaryopoiesis [38], while the chromatin accessibility, enhancer/promoter profiles, and transcriptional programs remain highly consistent. Changes in DNA methylation have been linked to induction of several megakaryocytic processes, including endomitosis, transcription factor expression and DNA affinity, and enhancer activity [39-42]. The requirement for de novo DNA methylation in megakaryopoiesis suggests that despite similar transcriptional and regulatory profiles with MPP, megakaryopoiesis does not constitute a "default" developmental program.

In contrast to megakaryopoiesis, erythropoiesis is associated with significant changes in the chromatin, enhancer/promoter, transcriptional, and methylation profiles. Our data suggest that, for most elements, these profiles are first detected at the level of the MEP, where the MEP ATAC-Seq profile clusters with that of mature erythroid cells. These data are consistent with the greater frequency of erythroid committed cells in the human MEP population [14]. Based on the transcriptional profiles, we propose that many cells in the mouse MEP population are in transition from multipotent progenitor cells (as evidenced by the similar transcriptional profiles) to erythroid committed cells. Our observations support this model and previous publications: ERY-specific AREs in CMP, but not LSK, contain TFBS associated with GATA-switching. However, in-depth single-cell analyses will be needed to test this hypothesis [43, 44].

The classic model of hematopoiesis is displayed as a hierarchy in which multipotent progenitors traverse a series of oligopotent and bipotent intermediates that progressively restrict lineage potential $[1,45,46]$. Recent studies, including single-cell transcriptional analyses and clonogenic assays, have identified a subset of megakaryocyte-primed progenitor cells in the MPP compartment that is proposed to give rise directly to megakaryocytes $[4,12,15,47,48]$. Our data are consistent with this model. Further down the hierarchy, multipotent progenitors give 


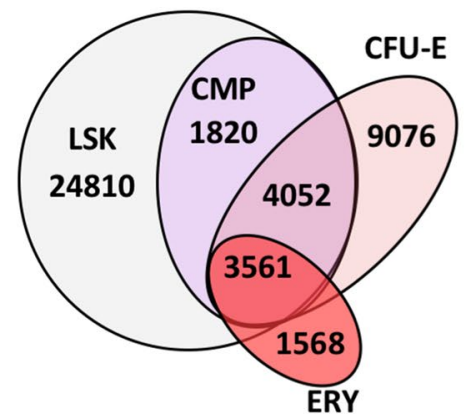

b

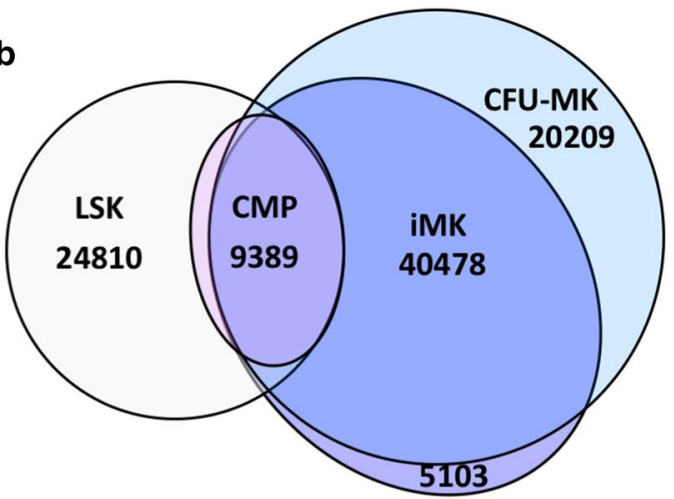

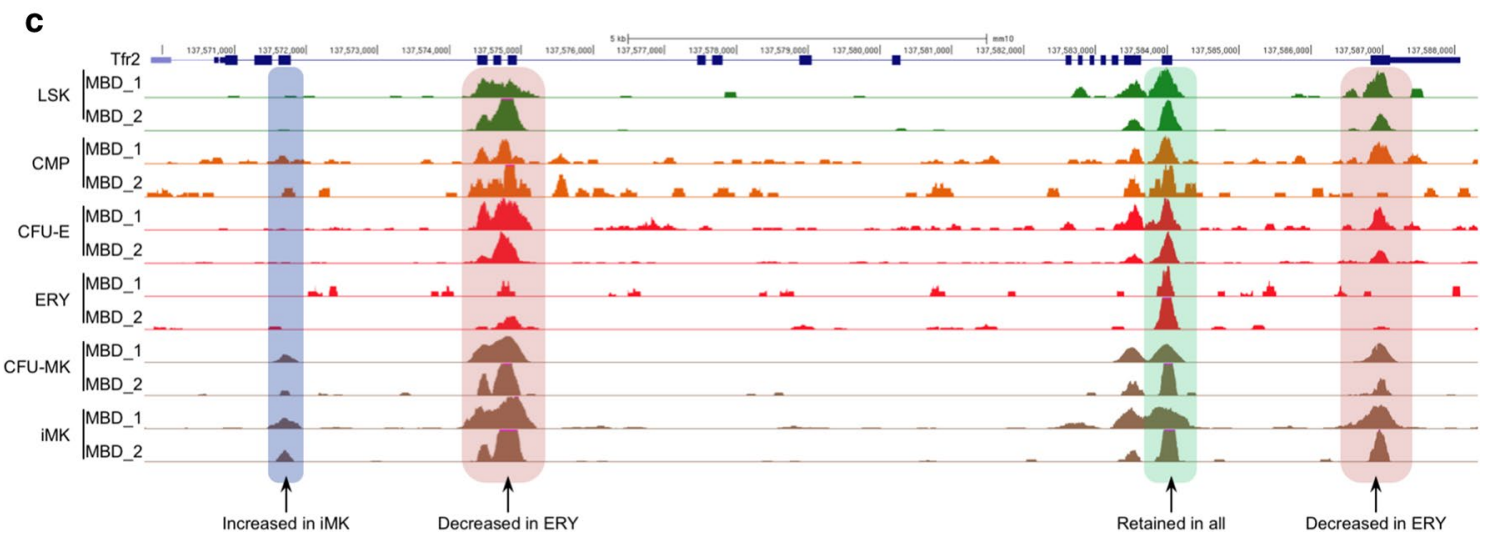

Fig. 8 Overlap of DNA methylation and chromatin-accessible AREs. a Establishment of DNA methylation in the erythroid lineage. $\mathbf{b}$ Establishment of DNA methylation in the megakaryocytic lineage. $\mathbf{c}$ The Tfr2 locus showing example regions of maintained, lost, and de novo DNA methylation during erythropoiesis and megakaryopoiesis

Table 5 Comparison of DNA methylation and AREs established during hematopoiesis

\begin{tabular}{lllll}
\hline ARE & \multicolumn{2}{l}{ DNA methylation } & & \\
\cline { 2 - 5 } & Mature cell & Committed progenitor & CMP & LSK \\
\hline ERY-specific & $43 / 2098(2.1 \%)[$ ERY] & $236 / 2054(11.5 \%)[C F U-E]$ & $64 / 1727(3.7 \%)$ & $207 / 1112(18.6 \%)$ \\
iMK-specific & $789 / 6386(12.4 \%)[$ iMK] & $1121 / 6286(17.8 \%)[C F U-M K]$ & $138 / 6068(2.3 \%)$ & $422 / 5385(7.8 \%)$ \\
\hline
\end{tabular}

Table 6 Comparison of DNA methylation and cell-specific regulatory elements in LSK

\begin{tabular}{lccc}
\hline ARE & \multicolumn{3}{l}{ DNA methylation in LSK } \\
\cline { 2 - 4 } & Active & Poised & Inactive \\
\hline ERY-specific & $28 / 144(19.4 \%)$ & $122 / 605(20.2 \%)$ & $56 / 335(16.7 \%)$ \\
iMK-specific & $126 / 1651(7.6 \%)$ & $189 / 2489(7.6 \%)$ & $86 / 978(8.8 \%)$ \\
\hline
\end{tabular}

rise to common myeloid progenitor (CMP) cells, then to the megakaryocyte-erythroid lineage at the MEP stage, and finally to either the megakaryocyte or erythroid lineages $[9,49]$. The existence of many of the cell types in this hierarchy is supported by the ability to grow colonies comprised of single or multiple lineages from single cells in semisolid medium in vitro. However, there are no reliable assays to culture both erythroid and megakaryocytic cells under the same conditions. Our data support models in which many cells within the classically defined MEP compartment have already become committed to one or the other lineage.

The data and analysis in this paper were generated as part of ValIdated Systematic IntegratiON of hematopoietic epigenomes (VISION) project, which aims to 
generate comprehensive catalogs of genomic regulatory elements in mouse and human hematopoietic cells and to conduct integrative statistical modeling and machine learning to predict regulatory interactions that are then validated by manipulating hematopoiesis at the genetic level. The data come both from efforts in the project laboratories, such as those generated for this paper, and also from other laboratories and consortia such as the Encyclopedia of DNA Elements (ENCODE) project and the NIH Roadmap Epigenomics Project [21-24]. These data are available at our website, usevision.org, for use by the larger community.

\section{Conclusion}

Our studies confirm that the majority of the epigenetic and transcriptional profiles found in the hematopoietic stem cell population are present in megakaryocytes, while the erythropoietic epigenetic and transcriptional programs are not established until erythroid-lineage commitment. By performing "bulk" analyses, we achieve greater depth of coverage that emphasizes the dominant characteristics of cell populations. In contrast, single-cell analyses can resolve finer distinctions between populations, but at a lower depth of coverage.

\section{Methods}

\section{Cell isolation}

All primary hematopoietic cell populations were enriched from 5-to-8-week-old C57BL6 male mice. LSK, CMP, MEP, GMP, CFU-E, ERY, CFU-MK, and iMK populations were collected from bone marrow. Following collection, samples were lineage-depleted using antibodies (Fig. 1) and conjugated to BioMag Goat anti-Rat IgG magnetic beads (cat \# 310007, Qiagen, Venlo, Netherlands). Lineage-depleted cells were then stained with fluorescently conjugated antibodies and sorted by flow cytometry on an FACSAria II (BD Biosciences). Neutrophils (NEU) and monocytes (MONO) were isolated directly from peripheral blood (PB). After Ficoll separation NEU were collected from the cell pellet following red cell lysis and MONO (Gr-1+Mac-1+ were sorted from the mononuclear fraction. All antibodies are listed in Table 7.

\section{RNA-Seq}

Cells were sorted into media and total RNA was extracted using TRIzol and the Ambion PureLink RNA Mini Kit (Life Technologies, cat\# 12183018), treated with DNase to remove genomic DNA using the DNAfree Kit (Life Technologies, cat\# AM1906). Sequencing libraries were then constructed from 100 ng of treated, total RNA using the ScriptSeq Complete Kit (Illumina cat\# BHMR1224) according to manufacturer's specifications. In brief, the RNA was subjected to rRNA depletion
Table 7 List of antibodies

\begin{tabular}{|c|c|c|}
\hline Antibody & Clone & Catalog number \\
\hline Rat a-CD4 & clone GK1.5 & $14-0041-86$ \\
\hline Rat a-IL7Ra & clone A7R34 & $14-1271-85$ \\
\hline Rat a-CD8 & clone 53-6.7 & $14-0081-86$ \\
\hline Rat a-Mac-1 & clone M1/70 & $14-0112-86$ \\
\hline Rat a-Gr-1 & clone RB6-8C5 & $14-5931-86$ \\
\hline Rat a-B220 & clone RA3-6B2 & $14-0452-86$ \\
\hline Rat a-Ter119 & clone TER-119 & $14-5921-85$ \\
\hline Rat a-Sca-1 (PE) & clone D7 & $12-5981-83$ \\
\hline Rat a-CD34 (FITC) & clone RAM34 & $11-0341-85$ \\
\hline Rat a-CD16/32 (PeCy7) & clone 93 & 25-0161-82 \\
\hline Rat IgG2b & clone eB149/10H5 & $14-4031-85$ \\
\hline Rat IgG1 & eBRG1 & $14-4301-85$ \\
\hline Rat lgG2a serum & eBR2a & $14-4321-85$ \\
\hline Rat a-cKit (APC) & clone 2B8 & 17-1171-83 \\
\hline Rat a-CD41 (PE) & clone eBioMWReg30 & $12-0411-83$ \\
\hline Rat a-CD61 (FITC) & clone 2C9.G3 & $11-0611-82$ \\
\hline Rat a-Sca-1 (PerCP-Cy5.5) & clone D7 & $45-5981-82$ \\
\hline Rat a-Ter119 (APC-780) & clone TER-119 & $47-5921-82$ \\
\hline Rat a-CD44 (eFluor450) & clone IM7 & $48-0441-82$ \\
\hline Rabbit a-H3K27ac & polyclonal & ab4729 \\
\hline Rabbit a-H3K4me1 & polyclonal & ab8895 \\
\hline Rat a-Mac-1 (eFluor450) & clone M1/70 & $48-0112-82$ \\
\hline Rat a-Gr-1 & clone RB6-8C6 & $45-5931-80$ \\
\hline
\end{tabular}

All antibodies were purchased from eBiosciences (San Diego, CA, USA)

using the Ribo-Zero removal reagents and fragmented. First-strand cDNA was then synthesized using a 5' tagged random hexamer and reverse transcription, followed by annealing of a 5' tagged, 3'-end blocked terminal-tagged oligo and second-strand synthesis. The Di-tagged cDNA fragments were purified, barcoded, and PCR-amplified for 15 cycles.

The size and quality of each library were then evaluated by Bioanalyzer 2100 (Agilent Technologies, Santa Clara, CA), and quantified using qPCR. Libraries were sequenced in paired-end mode on the NextSeq500 to generate $2 \times 75$ bp reads using Illumina-supplied kits as appropriate. The sequence reads were processed using the ENCODE3 long RNA-Seq pipeline (https://www. encodeproject.org/pipelines/ENCPL002LPE/). In brief, reads were mapped to the mouse genome ( $\mathrm{mm} 10$ assembly) using STAR v2.5.4 [50], followed by RSEM v1.3.0 [51] for gene quantifications. RNA-Seq was repeated to generate at least two biological replicates. Analyzed data are included as Additional file 4.

\section{ATAC-Seq}

Approximately 50,000 cells were collected by centrifugation at $600 \times \mathrm{g}$ for $10 \mathrm{~min}$ at $4{ }^{\circ} \mathrm{C}$. Cells were washed 
once with cold $1 \times$ PBS and centrifuged as above. Cells were lysed by gently pipetting to resuspend cell pellet in cold lysis buffer $(10 \mathrm{mM}$ Tris- $\mathrm{HCl} \mathrm{pH} 7.4,10 \mathrm{mM} \mathrm{NaCl}$, $3 \mathrm{mM} \mathrm{MgCl} 2,0.1 \%$ IGEPAL CA-630) and centrifuged as above. For each transposition reaction, cells were suspended in the following mix: $25 \mu \mathrm{l} 2 \mathrm{X}$ Tagment DNA buffer (Illumina cat\# FC-121-1030), $3 \mu \mathrm{l}$ Tn5 Transposase (Illumina cat\#FC-121-1030), $22 \mu \mathrm{l}$ nuclease-free $\mathrm{H} 2 \mathrm{O}$ and incubated for $30 \mathrm{~min}$ at $37^{\circ} \mathrm{C}$. Reactions were terminated by adding $5 \mu \mathrm{l}$ of $1 \%$ SDS solution and purified using SPRIselect beads (Beckman Coulter cat \#B23318) at a 1:1 ratio according to manufacturer's instructions, followed by a right side size selection using SPRIselect beads (Beckman Coulter cat \#B23318) at a 0:5 ratio according to manufacturer's instructions. Following purification, library fragments were amplified using $1 \times$ NEBnext PCR master mix and $1.25 \mu \mathrm{M}$ of custom Nextera PCR primers 1 and 2 [52] using the following PCR conditions: $72{ }^{\circ} \mathrm{C}$ for $5 \mathrm{~min} ; 98^{\circ} \mathrm{C}$ for $30 \mathrm{~s}$; and thermocycling at $98{ }^{\circ} \mathrm{C}$ for $10 \mathrm{~s}, 63{ }^{\circ} \mathrm{C}$ for $30 \mathrm{~s}$, and $72{ }^{\circ} \mathrm{C}$ for $1 \mathrm{~min}$. To reduce GC and size bias, the PCR was monitored using qPCR in order to stop amplification before saturation. To this end, libraries were amplified for five cycles, and then a $5 \mu \mathrm{l}$ aliquot of the PCR was removed and added $10 \mu \mathrm{l}$ of the PCR cocktail with SYBR Green at a final concentration of $0.6 \times$. This reaction was amplified for 20 cycles to determine the additional number of cycles needed for the remaining $45-\mu \mathrm{L}$ reaction. Libraries were amplified for a total of 17-19 cycles and then purified using AMPure XP beads (Beckman Coulter cat \#A63881) at a 1:1 ratio according to manufacturer's instructions. Constructed libraries were run on the Agilent Bioanalyzer 2100 (Agilent Technologies) using the 7500 DNA kit (cat\# 50671504) as appropriate to determine the average size and confirm the absence of unligated adaptors. Libraries were quantitated by qPCR using the Kapa SYBR FAST Universal kit (Kapa Biosystems) according to the Illumina's Sequencing Library qPCR Quantification Guide. Libraries were multiplexed and sequenced on the Illumina NextSeq500 using Illumina's kits and reagents as appropriate. ATAC-Seq was repeated to generate at least two biological replicates. Reads were mapped using Bowtie v1.0.0 [53], accessible regions identified with FSeq v1.85 [54], and peaks called using HOMER v1.0 [55]. To identify consensus peak sets, peaks from all datasets were combined and merged. Peaks present in both replicates within the same merged region were used for further analysis. Analyzed data are included as Additional file 5 .

\section{ChIP-Seq}

Approximately $20 \times 10^{6}$ cells were fixed in $0.4 \%$ formaldehyde (16\% methanol-free, Thermo Scientific) for $15 \mathrm{~min}$ before quenching in $125 \mathrm{mM}$ glycine for $5 \mathrm{~min}$.
Cells were washed in 2X PIC (Roche mini-tabs, 1 tab in $5 \mathrm{ml}=2 \mathrm{X}$ ) and stored at $-80^{\circ} \mathrm{C}$. Cells were resuspended in lysis buffer $(10 \mathrm{mM}$ Tris-HCl, pH8.0, $10 \mathrm{mM} \mathrm{NaCl}$, $0.2 \% \mathrm{NP} 40$ ) and sonicated (QSonica) to a size range of 200-500 bp as determined by electrophoresis. Immediately prior to immunoprecipitation, an aliquot was removed to be sequenced as a matched input control. Immunoprecipitation was performed using H3K27ac (Abcam ab4729) supplemented with $50 \mathrm{mM}$ sodium butyrate. DNA fragments were purified with AMPure XP beads (cat \# A63880, Agencourt, Pasadena, CA, USA) according to the manufacturer's protocol. Sequencing was performed at the NIH Intramural Sequencing Center on an Illumina HiSeq2500. Chromatin immunoprecipitations were repeated to generate four biological replicates. Sequence data were aligned to the $\mathrm{mm} 10$ genome using Bowtie v2.3.4 [56] and peak calling performed using SICER v1.1 [57]. Peaks present in all replicates were used for further analysis, as above.

We accessed indexing-first immunoprecipitation (iChIP) experimental data performed by Lara-Astiaso et al. [27] from Gene Omnibus (GSE60103). Sequencing reads from long- and short-term HSC (LT-HSC and ST-HSC, respectively) and multipotent progenitor cells were combined to generate "LSK" profiles from the iChIP data. These reads were aligned using Bowtie v2.3.4 and peak calling performed using MACS2 [58]. We combined LT-HSC, ST-HSC, and MPP peak sets to create the iChIP "LSK" population for downstream comparisons. For studies involving indexing-first chromatin immunoprecipitation data (iChIP), reads were accessed via GSE60103 (Lara-Astiaso et al. [27]), data aligned using Bowtie v2.3.4 and peaks-calling performed using MACS2 [58]. To create a set of LSK data, peaks were combined from the long-term HSCs, short-term HSCs, and multipotential progenitor sample sets. Analyzed data are included as Additional file 6 .

\section{MBD-Seq}

Genomic DNA was isolated from enriched cells with the QIAGEN Puregene kit and sonicated to 200- to 400-bp fragments (QSonica). MBD2 enrichment was performed with the Active Motif Methyl Collector kit. Approximately $1 \mu \mathrm{g}$ of sonicated genomic DNA was incubated with MBD2-His-conjugated protein and magnetic beads according to the manufacturer's protocol. After enrichment, both the methylated fraction and supernatant fractions were purified with QIAGEN DNA purification columns. Quantitative PCR amplification of the differentially methylated regions regulating the imprinting of Snrpn and Rasgrf1 and the unmethylated CpG island promoter of Actb was performed with SYBR Green PCR master mix (Applied Biosystems) and was used to 
validate the enrichment of methylated DNA using the MBD2-pull-down approach.

Two biological replicates of each enriched cell population and one supernatant sample per cell type were submitted for high-throughput sequencing analysis. Between 225 and 540 ng of MBD2-enriched DNA and $1 \mu \mathrm{g}$ of supernatant for each cell type were used to construct DNA libraries according to the Illumina protocol. The libraries were sequenced on the Illumina Genome Analyzer platform, and 36-bp single-end reads were used to uniquely identify the MBD2-bound fraction of the mouse genome. Sequenced reads were mapped to the mouse genome (UCSC assembly mm10) using Bowtie v2.3.4 [56]. Peaks for each cell type were called using the SigSeeker peak calling ensemble [59]. Reads from the matched supernatant were used as a control for each cell type. Replicate peaks that were called by two or more peak calling tools and overlapped by at least $100 \mathrm{bp}$ were considered for further analysis. Analyzed data are included as Additional file 6.

\section{Additional data analysis and visualization}

Permutation tests were performed using the $\mathrm{R}$ package regioneR v1.10.0. Transcription factor enrichment analyses were performed in HOMER v3.18 [55]. Graphs were created in PRISM v6.

\section{Additional files}

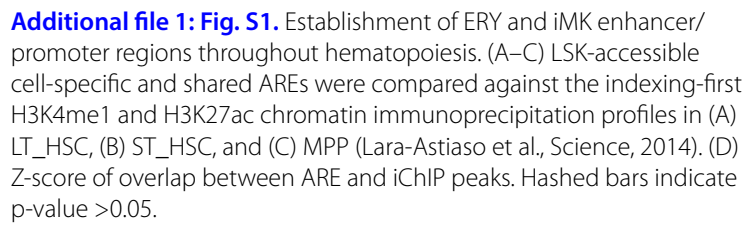

Additional file 1: Fig. S1. Establishment of ERY and iMK enhancer/ promoter regions throughout hematopoiesis. (A-C) LSK-accessible cell-specific and shared AREs were compared against the indexing-first H3K4me1 and H3K27ac chromatin immunoprecipitation profiles in (A) LT_HSC, (B) ST_HSC, and (C) MPP (Lara-Astiaso et al., Science, 2014). (D) Z-score of overlap between ARE and iChIP peaks. Hashed bars indicate p-value $>0.05$.

Additional file 2: Fig. S2. Locus-specific example of (epi) genomic correlations. (A) Erythroid specific induction of AREs and expression (S/c4a1) and constitutive AREs and expression (S/c25a39). RNA-Seq and ATAC-Seq are shown for all 10 cell types, and histone modifications for 5 cell types of most relevance. (B) Retention of AREs and expression from LSKs in MKs and loss in ERY. RNA-Seq and ATAC-Seq are shown for all 10 cell types, and histone modifications for 5 cell types of most relevance. Tracks are displayed on the mm10 genome.

Additional file 3. Enrichment scores of transcription factor binding sites in AREs. Unabridged list of enriched transcription factor binding sites in AREs. Scores are reported as q-values.

Additional file 4. RNA-Seq data with TPM calculations. Transcripts per million counts are included for each RNA-Seq replicate.

Additional file 5. Peak data from ATAC-Seq experiments. The presence of ATAC-Seq peaks in each cell type is indicated by a 1 (present) or 0 (not present)

Additional file 6. Peak data from ChIP-Seq and MBD-Seq experiments, Peaks from H3K27ac ChIP-Seq and MBD-Seq are presented in BED format. Note that due to file restrictions, MBD-Seq data for CFU-MK is included across two workbooks.

\section{Abbreviations}

HSC: hematopoietic stem cell; LSK: Lin ${ }^{-} \mathrm{Sca}^{+} \mathrm{Kit}^{+}$; MPP: multipotent progenitor: CMP: common myeloid progenitor; CLP: common lymphoid progenitor; GMP: granulocyte/macrophage progenitor; MEP: megakaryocyte/erythroid progenitor; CFU-MK: colony-forming unit (megakaryocyte); CFU-E: colony forming unit (erythrocyte); ERY: erythrocyte; MK: megakaryocyte; VISION: Valldated Systematic IntegratiON of hematopoietic epigenomes; ENCODE: Encyclopedia of DNA Elements; iMK: immature megakaryocyte; ATAC-Seq: assay for transposon-accessible chromatin followed by next-generation sequencing; RNA-Seq: next-generation sequencing of RNA; ChIP-Seq: chromatin immunoprecipitation followed by next-generation sequencing; MBD-Seq: methyl-binding protein immunoprecipitation followed by next-generation sequencing; TPM: transcripts per million count; PCA: principle component analysis; ARE: active regulatory element; iChIP: indexing-first chromatin immunoprecipitation; TSS: transcriptional start site; CEE: candidate enhancer element; CPE: candidate promoter element; TFBS: transcription factor binding site.

\section{Authors' contributions}

EFH generated H3K27ac ChIP-Seq data and analyzed, integrated, and interpreted next-generation sequencing experiments. CAK generated RNA-Seq and ATAC-Seq data. BG performed analyses and generated images regarding RNA-Seq and ATAC-Seq data. SMA performed flow cytometry analyses. JL generated MBD-Seq data. NISC performed sequencing for H3K27ac ChIP-Seq experiments. EFH, CAK, BG, JL, RCH, and DMB analyzed data and wrote the paper. All authors read and approved the final manuscript.

\section{Author details}

${ }^{1}$ NHGRI Hematopoiesis Section, GMBB, Bethesda, MD, USA. ${ }^{2}$ Pennsylvania State University, University Park, PA, USA. ${ }^{3}$ NHGRI Flow Cytometry Core, Bethesda, MD, USA. ${ }^{4} \mathrm{NHGRI}$, Rockville, MD, USA.

\section{Acknowledgements}

The authors would like to acknowledge Martha Kirby and Beth Psaila for their advice and assistance in preparation of this manuscript.

\section{Competing interests}

The authors declare that they have no competing interests.

\section{Availability of data and materials}

The datasets supporting the conclusions of this article are available in the VISION repository (http://usevision.org). iChIP data are available in the Gene Expression Omnibus repository, at https://www.ncbi.nlm.nih.gov/geo (accession number GSE60103, see citation below). Additionally, datasets supporting the conclusions of this article are included within the article and its additional files. Lara-Astiaso D, Weiner A, Lorenzo-Vivas E, et al. Chromatin state dynamics during blood formation. 2014. doi: https://doi.org/10.1126/science.1256271.

\section{Consent for publication}

Not applicable.

\section{Ethics approval and consent to participate}

Animal work followed the guidelines and policies and was approved by the Animal Care and Use Committee. The National Human Genome Research Institute is accredited by AAALAC International and is in accordance with the Public Health Service Policy for the Care and Use of Laboratory Animals.

\section{Funding}

This work was supported by the National Human Genome Research Institute intramural funds and the National Institute of Diabetes and Digestive and Kidney Diseases (Grant Number R24DK106766-01A1).

\section{Publisher's Note}

Springer Nature remains neutral with regard to jurisdictional claims in published maps and institutional affiliations.

Received: 14 April 2018 Accepted: 21 May 2018

Published online: 28 May 2018 


\section{References}

1. Orkin SH, Zon LI. Hematopoiesis: an evolving paradigm for stem cell biology. Cell. 2008;132:631-44.

2. Seita J, Weissman IL. Hematopoietic stem cell: self-renewal versus differentiation. Wiley Interdiscip Rev Syst Biol Med. 2010;2:640-53.

3. Krause DS. Regulation of hematopoietic stem cell fate. Oncogene. 2002;21:3262.

4. Velten L, Haas SF, Raffel S, Blaszkiewicz S, Islam S, Hennig BP, Hirche C, Lutz C, Buss EC, Nowak D, et al. Human haematopoietic stem cell lineage commitment is a continuous process. Nat Cell Biol. 2017;19:271.

5. Bresnick EH, Lee HY, Fujiwara T, Johnson KD, Keles S. GATA switches as developmental drivers. J Biol Chem. 2010;285:31087-93.

6. Doré LC, Crispino JD. Transcription factor networks in erythroid cell and megakaryocyte development. Blood. 2011;118:231-9.

7. Ghamari A, Pregerning G, Fraenkel E, Cantor AB. GATA factor switching during erythroid differentiation is facilitated by FBW7 mediated clearance of GATA2. Blood. 2016;128:1479.

8. Pimkin M, Kossenkov AV, Mishra T, Morrissey CS, Wu W, Keller CA, Blobel GA, Lee D, Beer MA, Hardison RC, Weiss MJ. Divergent functions of hematopoietic transcription factors in lineage priming and differentiation during erythro-megakaryopoiesis. Genome Res. 2014;24:1932-44.

9. Debili N, Coulombel L, Croisille L, Katz A, Guichard J, Breton-Gorius J. Characterization of a bipotent erythro-megakaryocytic progenitor in human bone marrow. Blood. 1996;88:1284-96.

10. Woolthuis CM, Park CY. Hematopoietic stem/progenitor cell commitment to the megakaryocyte lineage. Blood. 2016;127:1242-8.

11. Huang $\mathrm{H}$, Cantor $\mathrm{AB}$. Common features of megakaryocytes and hematopoietic stem cells: What's the connection? J Cell Biochem. 2009;107:857-64.

12. Sanjuan-Pla A, Macaulay IC, Jensen CT, Woll PS, Luis TC, Mead A, Moore S, Carella C, Matsuoka S, Jones TB, et al. Platelet-biased stem cells reside at the apex of the haematopoietic stem-cell hierarchy. Nature. 2013;502:232-6.

13. Pinho S, Marchand T, Yang E, Wei Q, Nerlov C, Frenette PS. Lineage-biased hematopoietic stem cells are regulated by distinct niches. Dev Cell. 2018:44:634-41

14. Psaila B, Barkas N, Iskander D, Roy A, Anderson S, Ashley N, Caputo VS, Lichtenberg J, Loaiza S, Bodine DM. Single-cell profiling of human megakaryocyte-erythroid progenitors identifies distinct megakaryocyte and erythroid differentiation pathways. Genome Biol. 2016;17:83.

15. Notta F, Zandi S, Takayama N, Dobson S, Gan OI, Wilson G, Kaufmann KB, McLeod J, Laurenti E, Dunant CF, et al. Distinct routes of lineage development reshape the human blood hierarchy across ontogeny. Science. 2016;351:aab2116.

16. Wegman-Ostrosky T, Savage SA. The genomics of inherited bone marrow failure: from mechanism to the clinic. Br J Haematol. 2017:177:526-42.

17. Songdej N, Rao AK. Hematopoietic transcription factor mutations: important players in inherited platelet defects. Blood. 2017;129:2873.

18. Duployez N, Lejeune S, Renneville A, Preudhomme C. Myelodysplastic syndromes and acute leukemia with genetic predispositions: a new challenge for hematologists. Expert Rev Hematol. 2016;9:1189-202.

19. Dailey HA, Meissner PN. Erythroid heme biosynthesis and its disorders. Cold Spring Harb Perspect Med. 2013:3:a011676.

20. Donadieu J, Beaupain B, Fenneteau O, Bellanné-Chantelot C. Congenital neutropenia in the era of genomics: classification, diagnosis, and natural history. Br J Haematol. 2017;179:557-74.

21. Roadmap Epigenomics C, Kundaje A, Meuleman W, Ernst J, Bilenky M, Yen A, Heravi-Moussavi A, Kheradpour P, Zhang Z, Wang J, et al. Integrative analysis of 111 reference human epigenomes. Nature. 2015;518:317-30.

22. The ENCODE Project Consortium. An integrated encyclopedia of DNA elements in the human genome. Nature. 2012;489:57-74.

23. The ENCODE Project Consortium. The ENCODE (ENCyclopedia Of DNA Elements) Project. Science. 2004;306:636

24. Hong EL, Sloan CA, Chan ET, Davidson JM, Malladi VS, Strattan JS, Hitz BC, Gabdank I, Narayanan AK, Ho M, et al. Principles of metadata organization at the ENCODE data coordination center. Database J Biol Databases Curation. 2016;2016:baw001.

25. Creyghton MP, Cheng AW, Welstead GG, Kooistra T, Carey BW, Steine EJ, Hanna J, Lodato MA, Frampton GM, Sharp PA, et al. Histone H3K27ac separates active from poised enhancers and predicts developmental state. Proc Natl Acad Sci USA. 2010:107:21931-6.
26. Gel B, Díez-Villanueva A, Serra E, Buschbeck M, Peinado MA, Malinverni $R$. regioneR: an $R /$ Bioconductor package for the association analysis of genomic regions based on permutation tests. Bioinformatics. 2016;32:289-91.

27. Lara-Astiaso D, Weiner A, Lorenzo-Vivas E, Zaretsky I, Jaitin DA, David E, Keren-Shaul H, Mildner A, Winter D, Jung S, et al. Chromatin state dynamics during blood formation. Science. 2014;345:943-9.

28. Dogan N, Wu W, Morrissey CS, Chen K-B, Stonestrom A, Long M, Keller CA Cheng $Y$, Jain $D$, Visel $A$, et al. Occupancy by key transcription factors is a more accurate predictor of enhancer activity than histone modifications or chromatin accessibility. Epigenet Chromatin. 2015;8:16.

29. Lichtenberg J, Bodine DM. Evaluation of peak calling approaches for genome-wide MBD2-based methyl-seq profiling. In: 2017 IEEE 7th international conference on computational advances in bio and medical sciences (ICCABS), 19-21 Oct 2017; 2017. pp. 1-6.

30. Nishikii H, Kanazawa Y, Umemoto T, Goltsev Y, Matsuzaki Y, Matsushita K. Unipotent megakaryopoietic pathway bridging hematopoietic stem cells and mature megakaryocytes. Stem Cells. 2015;33:2196-207.

31. Nishikii H, Kurita N, Chiba S. The road map for megakaryopoietic lineage from hematopoietic stem/progenitor cells. Stem Cells Transl Med. 2017;6:1661-5.

32. Macaulay IC, Svensson V, Labalette C, Ferreira L, Hamey F, Voet T. Singlecell RNA-sequencing reveals a continuous spectrum of differentiation in hematopoietic cells. Cell Rep. 2016;14:966-77.

33. Waddington $\mathrm{CH}$. The strategy of the genes a discussion of some aspects of theoretical biology. London: Allen \& Unwin; 1957.

34. Quesenberry PJ, Goldberg L, Aliotta J, Dooner M. Marrow hematopoietic stem cells revisited: they exist in a continuum and are not defined by standard purification approaches; then there are the microvesicles. Front Oncol. 2014;4:56

35. Podobinska M, Szablowska-Gadomska I, Augustyniak J, Sandvig I, Sandvig A, Buzanska L. Epigenetic modulation of stem cells in neurodevelopment the role of methylation and acetylation. Front Cell Neurosci. 2017;11:23

36. Cheng $Y$, Xie N, Jin P, Wang T. DNA methylation and hydroxymethylation in stem cells. Cell Biochem Funct. 2015;33:161-73.

37. Tsumagari K, Baribault C, Terragni J, Varley KE, Gertz J, Pradhan S, Badoo M, Crain CM, Song L, Crawford GE, et al. Early de novo DNA methylation and prolonged demethylation in the muscle lineage. Epigenetics. 2013;8:317-32.

38. Lichtenberg J, Heuston EF, Anderson SM, Keller CA, Hardison RC, Bodine DM, NIH Intramural Sequencing Center. De novo DNA methylation is associated with granulopoiesis and megakaryopoiesis but not erythropoiesis. Blood. 2016;128:3868.

39. Farlik M, Halbritter F, Müller F, Choudry Fizzah A, Ebert $P$, Klughammer J, Farrow S, Santoro A, Ciaurro V, Mathur A, et al. DNA methylation dynamics of human hematopoietic stem cell differentiation. Cell Stem Cell. 2016:19:808-22.

40. Ji H, Ehrlich LIR, Seita J, Murakami P, Doi A, Lindau P, Lee H, Aryee MJ, Irizarry RA, Kim K, et al. A comprehensive methylome map of lineage commitment from hematopoietic progenitors. Nature. 2010;467:338-42.

41. Izzi B, Pistoni M, Cludts K, Akkor P, Lambrechts D, Verfaillie C, Verhamme P, Freson K, Hoylaerts MF. Allele-specific DNA methylation capacitates PEAR1 enhancer activity. Blood. 2016:128:1003-12.

42. Lichtenberg J, Heuston EF, Keller CA, Hardison RC, Bodine DM. Comparison of expression and epigenetic profiles in human and mouse erythropoiesis and megakaryopoiesis using a systems biology model. Blood. 2015; 126:2383

43. Litzenburger UM, Buenrostro JD, Wu B, Shen Y, Sheffield NC, Kathiria A, GreenleafWJ, Chang HY. Single-cell epigenomic variability reveals functional cancer heterogeneity. Genome Biol. 2017;18:15.

44. Hogart A, Lichtenberg J, Ajay SS, Anderson S, Margulies EH, Bodine DM. Genome-wide DNA methylation profiles in hematopoietic stem and progenitor cells reveal overrepresentation of ETS transcription factor binding sites. Genome Res. 2012:22:1407-18.

45. Till JE, McCulloch EA. Hemopoietic stem cell differentiation. Biochim Biophys Acta (BBA) Rev Cancer. 1980;605:431-59.

46. Spangrude GJ, Heimfeld S, Weissman IL. Purification and characterization of mouse hematopoietic stem cells. Science. 1988;241:58.

47. Haas S, Hansson J, Klimmeck D, Loeffler D, Velten L, Uckelmann H. Inflammation-induced emergency megakaryopoiesis driven by hematopoietic stem cell-like megakaryocyte progenitors. Cell Stem Cell. 2015;17:422-34. 
48. Yamamoto R, Morita Y, Ooehara J, Hamanaka S, Onodera M, Rudolph KL. Clonal analysis unveils self-renewing lineage-restricted progenitors generated directly from hematopoietic stem cells. Cell. 2013;154:1112-26.

49. Weissman IL, Anderson DJ, Gage F. Stem and progenitor cells: origins, phenotypes, lineage commitments, and transdifferentiations. Annu Rev Cell Dev Biol. 2001;17:387-403.

50. Dobin A, Davis CA, Schlesinger F, Drenkow J, Zaleski C, Jha S, Batut $P$, Chaisson M, Gingeras TR. STAR: ultrafast universal RNA-seq aligner. Bioinformatics. 2012;29:15-21.

51. Li B, Dewey C. RSEM: accurate transcript quantification from RNA-Seq data with or without a reference genome. BMC Bioinform. 2011;12:323.

52. Buenrostro JD, Giresi PG, Zaba LC, Chang HY, Greenleaf WJ. Transposition of native chromatin for fast and sensitive epigenomic profiling of open chromatin, DNA-binding proteins and nucleosome position. Nat Methods. 2013;10:1213-8.

53. Langmead B, Trapnell C, Pop M, Salzberg SL. Ultrafast and memoryefficient alignment of short DNA sequences to the human genome. Genome Biol. 2009;10:R25.
54. Boyle AP, Guinney J, Crawford GE, Furey TS. F-Seq: a feature density estimator for high-throughput sequence tags. Bioinformatics. 2008:24:2537-8.

55. Heinz S, Benner C, Spann N, Bertolino E, Lin YC, Laslo P, Cheng JX, Murre C, Singh $\mathrm{H}$, Glass CK. Simple combinations of lineage-determining transcription factors prime cis-regulatory elements required for macrophage and B cell identities. Mol Cell. 2010;38:576-89.

56. Langmead B, Salzberg SL. Fast gapped-read alignment with Bowtie 2. Nat Methods. 2012:9:357

57. Zang C, Schones DE, Zeng C, Cui K, Zhao K, Peng W. A clustering approach for identification of enriched domains from histone modification ChIP-Seq data. Bioinformatics. 2009;25:1952-8.

58. Zhang Y, Liu T, Meyer CA, Eeckhoute J, Johnson DS, Bernstein BE, Nusbaum C, Myers RM, Brown M, Li W, Liu XS. Model-based analysis of ChIP-Seq (MACS). Genome Biol. 2008;9:R137.

59. Lichtenberg J, Elnitski L, Bodine DM. SigSeeker: a peak-calling ensemble approach for constructing epigenetic signatures. Bioinformatics. 2017;33:2615-21
Ready to submit your research? Choose BMC and benefit from:

- fast, convenient online submission

- thorough peer review by experienced researchers in your field

- rapid publication on acceptance

- support for research data, including large and complex data types

- gold Open Access which fosters wider collaboration and increased citations

- maximum visibility for your research: over $100 \mathrm{M}$ website views per year

At BMC, research is always in progress.

Learn more biomedcentral.com/submissions 